\title{
Computing three-point functions for short operators
}

\author{
Till Bargheer ${ }^{a, b}$ Joseph A. Minahan ${ }^{c}$ and Raul Pereira ${ }^{c}$ \\ ${ }^{a}$ School of Natural Sciences, The Institute for Advanced Study, \\ Einstein Drive, Princeton, NJ 08540, U.S.A. \\ ${ }^{b}$ DESY Theory Group, DESY Hamburg, \\ Notkestraße 85, D-22603 Hamburg, Germany \\ ${ }^{c}$ Department of Physics and Astronomy, Uppsala University, \\ Box 520, SE-751 20 Uppsala, Sweden \\ E-mail: bargheer@ias.edu, joseph.minahan, raul.pereira@physics.uu.se
}

ABSTRACT: We compute the three-point structure constants for short primary operators of $\mathcal{N}=4$ super Yang-Mills theory to leading order in $1 / \sqrt{\lambda}$ by mapping the problem to a flat-space string theory calculation. We check the validity of our procedure by comparing to known results for three chiral primaries. We then compute the three-point functions for any combination of chiral and non-chiral primaries, with the non-chiral primaries all dual to string states at the first massive level. Along the way we find many cancellations that leave us with simple expressions, suggesting that integrability is playing an important role.

KeYwords: Supersymmetric gauge theory, Extended Supersymmetry, AdS-CFT Correspondence

ARXIV EPRINT: 1311.7461 


\section{Contents}

1 Introduction 1

2 Review of previous results 5

3 Three-point functions $\quad 10$

3.1 Three chiral primaries 11

$\begin{array}{ll}3.2 & \text { Two chiral primaries } \\ 3.3 & \text { One }\end{array}$

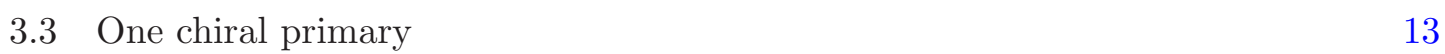

$\begin{array}{lll}3.4 & \text { Zero chiral primaries } & 14\end{array}$

$\begin{array}{llr}4 & \text { Discussion } & 14\end{array}$

$\begin{array}{lr}\text { A Bosonization setup and OPE's } & 16\end{array}$

$\begin{array}{lr}\text { B Primary vertex operators } & 19\end{array}$

$\begin{array}{ll}\text { B.1 Massless vertex operators } & 19\end{array}$

$\begin{array}{ll}\text { B.2 Massive vertex operators } & 20\end{array}$

B.3 Untwisting 21

C Vertex function contractions $\quad 22$

$\begin{array}{lll}\text { C.1 Fermion VEVs } & 22\end{array}$

C.2 Contractions with three chiral primaries 23

C.3 Contractions with two chiral primaries 24

$\begin{array}{ll}\text { C.4 Contractions with one chiral primary } & 26\end{array}$

$\begin{array}{ll}\text { C.5 Contractions with zero chiral primaries } & 29\end{array}$

D Mixed correlators $\quad 32$

\section{Introduction}

It is now possible, at least in principle, to compute the dimensions of single-trace local operators in planar $\mathcal{N}=4$ super Yang-Mills for any value of the 't Hooft coupling $\lambda$. The ability to do this relies on the underlying integrability of the theory [1]. One of the most impressive results is the numerical computation of the dimension, $\Delta_{\mathrm{K}}$, of the Konishi operator, $\mathcal{O}_{\mathrm{K}}=\operatorname{Tr} \Phi^{I} \Phi_{I}$. Starting at weak coupling and interpolating to very large values of $\lambda$, one finds extremely precise results $[2,3]$. What is clear from these studies is that for large values of $\lambda, \Delta_{\mathrm{K}}$ asymptotes to

$$
\Delta_{\mathrm{K}}=2 \lambda^{1 / 4}-2+\frac{2}{\lambda^{1 / 4}}+\ldots \quad \lambda \gg 1,
$$


a very satisfying result since the leading behavior was predicted well beforehand based on a flat-space approximation of a string on $\mathrm{AdS}_{5} \times \mathrm{S}^{5}$ [4]. The next two terms in this series can also be motivated by semi-classical string theory computations [5-10], or by analyzing the algebraic curve [11, 12], or by a mini-superspace approach on the string world-sheet [13].

To fully solve planar $\mathcal{N}=4 \mathrm{SYM}$ it is also necessary to know the three-point functions between local operators. Compared to the spectrum, the results here are rather limited. However, there are indications that integrability will play a crucial role in determining the structure constants. In particular, there have been important results at low orders of perturbation theory that crucially rely on the integrable structure in the theory [14-18]. Impressively, in [15] it was shown that the weak-coupling three-point function for two semi-classical operators (with $\Delta \sim \sqrt{\lambda}$ ) and one short BPS operator, was consistent with the strong-coupling results derived from a semi-classical string calculation in [19, 20].

Based on our experience with two-point functions, it will be much more difficult to compare weak and strong coupling results if the operators in the three-point functions are all short. ${ }^{1}$ This is because finite-size effects are expected to become important at strong-coupling and so all of the machinery of the thermodynamic Bethe ansatz will likely be needed. Nonetheless, as with $\Delta_{\mathrm{K}}$, it will be very useful to know the strong-coupling target. In other words, analogous to the GKP result in [4], we wish to know the leading strong-coupling behavior for such three-point functions.

In this paper we continue the study of three-point functions for short operators at level one. The methods we describe take advantage of the fact that at large Yang-Mills coupling the string theory target space has small curvature and so one can approximate the vertex operators with flat-space vertex operators [21]. The curvature still plays a role as it determines how the states propagate in from the boundary. The accompanying Witten diagrams have an intersection point that should be integrated over, but for short operators with a large dimension the integral is dominant over a small region of the bulk ${ }^{2}$ where one can ignore the curvature as well as the Ramond-Ramond flux. Within that region, one can then use flat space vertex operators to compute the couplings that determine the part of the three-point function not determined solely by the dimensions of the operators.

The challenge is to find the flat-space vertex operators and then compute the threepoint function with those respective operators. In [21] it was described how to find such operators. In particular, it was argued that the relevant operators satisfy a twisted version of $Q_{\mathrm{L}}=i Q_{\mathrm{R}}$, where $Q_{\mathrm{L}, \mathrm{R}}$ are the left and right ten-dimensional supercharges in flat space. It was shown that this condition on the supercharges maps back to the AdS condition that $S=0$, where $S$ represents all superconformal charges. We impose this AdS condition since the three operators are assumed to be primaries. Consequently, the operators are necessarily combinations of NS-NS and R-R scalars.

Since the Konishi operator is primary but not chiral primary, its string dual is a massive state. In the large coupling limit, the dimensions of scalar operators with no $R$-charges have dimensions that are approximately $\Delta \approx 2 \sqrt{n} \lambda^{1 / 4}$ corresponding to the energies of stringy

\footnotetext{
${ }^{1}$ By "short" we mean local single-trace operators whose dual string states are short compared to the radii of curvature in $\mathrm{AdS}_{5} \times \mathrm{S}^{5}$. We do not mean operators that are in short super-multiplets.

${ }^{2}$ The same thing leads to the focusing of wave-packets for AdS $S$-matrices [22-24].
} 
modes when $\alpha^{\prime}=1 / \sqrt{\lambda}[4]$. There is now overwhelming evidence that the Konishi operator approaches one of these stringy modes with $n=1[25,26]$. Hence, the three-point function for three Konishi operators at strong coupling can be well-approximated by determining the three-point amplitude for three massive string states in flat IIB string theory.

Unfortunately, the vertex operators are quite complicated, even at the lowest massive level, and to the best of our knowledge a three-point function involving three massive IIB vertex operators has never been computed. ${ }^{3}$ In this paper we will do just that for three scalar operators, although they are not scalars in the ten-dimensional theory. We will also compute three-point functions involving one, two and three chiral primaries, which are dual to massless string states, and whose dimensions equal the sum of their $R$-charges. In the case of three chiral primaries we will compare our result to the supergravity result in [28], showing that our procedure is consistent with their result. The correlator with one chiral primary whose $R$-charge $J$ satisfies $J \ll \lambda^{1 / 4}$ is of significance because it comes with an exponential suppression factor that depends on $J$ and not on the dimensions of the two non-chiral primaries.

The full three-point function of the operators also relies on overlap integrals on the $\mathrm{S}^{5}$. For chiral primaries, it is necessary to assume that the $R$-charges are large so that their corresponding dimensions are large. This will result in wave-functions that are strongly peaked on $\mathrm{S}^{5}$. However, for non-chiral primaries the dimensions are already large in the strong-coupling limit and there is otherwise no reason to assume large $R$-charges for these operators, ${ }^{4}$ provided that the total $R$-charge in the three-point function is conserved. In any case we will give expressions for three-point functions for general $J$ values, but also give results for when the $R$-charges of the non-chiral primaries are zero, which happens for three Konishi operators.

The three-point function for three scalar operators in $d$ space-time dimensions with scaling dimensions $1 \ll \Delta_{i} \ll \sqrt{\lambda}$ is given by

$$
\left\langle\mathcal{O}_{\Delta_{1}}\left(x_{1}^{\mu}\right) \mathcal{O}_{\Delta_{2}}\left(x_{2}^{\mu}\right) \mathcal{O}_{\Delta_{3}}\left(x_{3}^{\mu}\right)\right\rangle=\frac{\mathcal{C}_{123}}{\left|x_{12}\right|^{\Delta_{1}+\Delta_{2}-\Delta_{3}}\left|x_{23}\right|^{\Delta_{2}+\Delta_{3}-\Delta_{1}}\left|x_{31}\right|^{\Delta_{3}+\Delta_{1}-\Delta_{2}}},
$$

where $[21,29]$

$$
\mathcal{C}_{123} \approx \frac{\pi^{\frac{2-d}{4}}}{4} \frac{\left(\Delta_{1} \Delta_{2} \Delta_{3}\right)^{d / 4}}{\left(\alpha_{1} \alpha_{2} \alpha_{3} \Sigma^{d+1}\right)^{1 / 2}} \frac{\alpha_{1}^{\alpha_{1}} \alpha_{2}^{\alpha_{2}} \alpha_{3}^{\alpha_{3}} \Sigma^{\Sigma}}{\Delta_{1}^{\Delta_{1}} \Delta_{2}^{\Delta_{2}} \Delta_{3}^{\Delta_{3}}} \mathcal{G}_{123}
$$

and

$$
\begin{aligned}
\alpha_{1} & =\frac{1}{2}\left(\Delta_{2}+\Delta_{3}-\Delta_{1}\right), \quad \alpha_{2}=\frac{1}{2}\left(\Delta_{3}+\Delta_{1}-\Delta_{2}\right), \quad \alpha_{3}=\frac{1}{2}\left(\Delta_{1}+\Delta_{2}-\Delta_{3}\right), \\
\Sigma & =\frac{1}{2}\left(\Delta_{1}+\Delta_{2}+\Delta_{3}\right)=\alpha_{1}+\alpha_{2}+\alpha_{3} .
\end{aligned}
$$

\footnotetext{
${ }^{3}$ The form of superstring three-point amplitudes are constrained by supersymmetric Ward and factorization identities [27]. It would be interesting to understand to what extent such identities can determine the R-charge-dependent normalization factors that we compute in this work.

${ }^{4}$ We thank E. Witten for a discussion on this point.
} 
The coupling $\mathcal{G}_{123}$ is given by

$$
\mathcal{G}_{123}=\frac{8 \pi}{g_{\mathrm{c}}^{2} \alpha^{\prime}}\left\langle V_{k_{1}} V_{k_{2}} V_{k_{3}}\right\rangle\left\langle\psi_{J_{1}} \psi_{J_{2}} \psi_{J_{3}}\right\rangle,
$$

which includes the contribution of the overlap integral $\left\langle\psi_{J_{1}} \psi_{J_{2}} \psi_{J_{3}}\right\rangle$ on $S^{5}$. The closed string coupling $g_{\mathrm{c}}$ and the inverse string tension are given under the AdS/CFT dictionary as $g_{\mathrm{c}}=\pi^{3 / 2} / N$ and $\alpha^{\prime}=1 / \sqrt{\lambda}$.

In this paper we will find for three level-one states with small $R$-charges $J_{i} \ll \lambda^{1 / 4}$ that the flat-space vertex operators satisfy

$$
\left\langle V_{k_{1}} V_{k_{2}} V_{k_{3}}\right\rangle=g_{\mathrm{c}}^{3} \frac{3^{8}}{2^{9}}+\mathcal{O}\left(J_{i}^{2} \lambda^{-1 / 2}\right)
$$

Hence the result for the correlator of three short operators with small $R$-charges reads

$$
\mathcal{C}_{123} \approx \frac{1}{N} \frac{3^{11 / 2} \pi^{2}}{32} \lambda^{1 / 4}\left(\frac{3}{4}\right)^{3 \Delta / 2}\left\langle\psi_{J_{1}} \psi_{J_{2}} \psi_{J_{3}}\right\rangle .
$$

For vanishing $R$-charges $J_{i}$ we have that $\left\langle\psi_{J_{1}} \psi_{J_{2}} \psi_{J_{3}}\right\rangle=\pi^{-3 / 2}$. Including the finite correction term in (1.1) [5], we can thus reëxpress (1.7) for three Konishi operators as

$$
\mathcal{C}_{123} \approx \frac{1}{N} 64 \pi^{1 / 2} \lambda^{1 / 4}\left(\frac{3}{4}\right)^{3 \lambda^{1 / 4}+5 / 2} .
$$

In the intermediate steps the individual contributions to (1.6) involve larger prime factors, but after combining the different parts we end up with a much simpler expression. This suggests that there is an underlying symmetry playing an important role.

For the case of one chiral primary and two primaries at level one, we find that

$$
\mathcal{C}_{123} \approx \frac{1}{N} \pi^{2} \lambda^{1 / 4} 2^{-J_{3}}\left\langle\psi_{J_{1}} \psi_{J_{2}} \psi_{J_{3}}\right\rangle
$$

where $1 \ll J_{3} \ll \lambda^{1 / 4}$ is the magnitude of the $R$-charge of the chiral primary, and $\left\langle\psi_{J_{1}} \psi_{J_{2}} \psi_{J_{3}}\right\rangle$ depends on the distribution of $R$-charge on the level-one states. For $J_{1}=0$, $J_{2}=J_{3}=J$, the correlator becomes

$$
\mathcal{C}_{123} \approx \frac{1}{N} \pi^{1 / 2} \lambda^{1 / 4} 2^{-J}
$$

Note that $\mathcal{C}_{123}$ is exponentially suppressed if all $\Delta_{i} \gg 1$. However, if one of the operators is a chiral primary with $\Delta=J$ and $J$ is much smaller than the other two dimensions, then the suppression factor is only by $2^{-J}$.

The rest of this paper is organized as follows: in section 2 we review some results of [21]. In section 3, which is the main part of this article, we compute three-point amplitudes for three string states where zero, one, two or three are at the first massive level. We then use these to reach the main results in (1.7) and (1.9). The partial amplitudes involve combinations of three NS-NS scalars or one NS-NS scalar and two R-R scalars. The states are not Lorentz scalars in the full ten-dimensional flat space, but instead are twisted 
versions of these states. However, for $J=0$ they remain scalars in the two reduced five-dimensional spaces which are the flattened versions of $\mathrm{AdS}_{5}$ and $\mathrm{S}^{5}{ }^{5}$ Section 4 contains our conclusions and discussion for various extensions of these results. Many technical details are contained in several appendices.

\section{Review of previous results}

In this section we collect some relevant results from [21].

We assume that the operators are short operators in the sense that their sizes are much smaller than the $\mathrm{AdS}_{5}$ and $\mathrm{S}^{5}$ radii. Hence at scales comparable to this radius the strings appear point-like. The three-point correlator is then closely related to the computation of a three-point correlator using Witten diagrams [30, 31].

In the Witten diagram one integrates over all possible intersection points, but in the semiclassical approximation the Witten diagrams are dominated by peaks along the particles' classical trajectories in the $\mathrm{AdS}_{5} \times \mathrm{S}^{5}$ background. The intersection point is calculable $[21,29]$, and after including the contributions of the fluctuations one finds the coefficient in front of $\mathcal{G}_{123}$ in (1.3). For large $R$-charges $J_{i} \gg 1$ we can also use a semiclassical approximation for the $\mathrm{S}^{5}$ overlap integrals $\left\langle\psi_{J_{1}} \psi_{J_{2}} \psi_{J_{3}}\right\rangle[21,32]$. In this approximation we assume that the $R$-charges are each highest weight with respect to some basis and satisfy $\overrightarrow{J_{1}}+\overrightarrow{J_{2}}+\overrightarrow{J_{3}}=0$. Then one finds

$$
\left\langle\psi_{J_{1}} \psi_{J_{2}} \psi_{J_{3}}\right\rangle \approx \frac{1}{2 \pi^{2}} \frac{\left(J_{1} J_{2} J_{3}\right)^{3 / 2}}{\left(\tilde{\alpha}_{1} \tilde{\alpha}_{2} \tilde{\alpha}_{3} \tilde{\Sigma}^{5}\right)^{1 / 2}},
$$

where

$$
\tilde{\Sigma}=\frac{J_{1}+J_{2}+J_{3}}{2}, \quad \tilde{\alpha}_{1}=\frac{J_{2}+J_{3}-J_{1}}{2}, \quad \tilde{\alpha}_{2}=\frac{J_{3}+J_{1}-J_{2}}{2}, \quad \tilde{\alpha}_{3}=\frac{J_{1}+J_{2}-J_{3}}{2} .
$$

For later reference, these can be written in terms of the standard spherical harmonic coefficients $C_{I_{1} \ldots I_{J}}^{J}$ in $[28]$ as

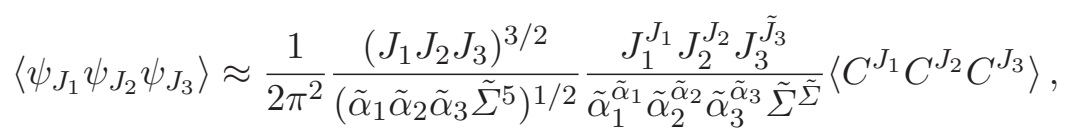

where

$$
\left\langle C^{J_{1}} C^{J_{2}} C^{J_{3}}\right\rangle \approx \frac{\tilde{\alpha}_{1}^{\tilde{\alpha}_{1}} \tilde{\alpha}_{2}^{\tilde{\alpha}_{2}} \tilde{\alpha}_{3}^{\tilde{\alpha}_{3}} \tilde{\Sigma}^{\tilde{\Sigma}}}{J_{1}^{J_{1}} J_{2}^{J_{2}} J_{3}^{J_{3}}}
$$

The classical trajectories come with constants of motion which are determined by the $R$-charges, the spins, the dimensions and by the positions of the operators on the boundary. The constants of the motion can be formulated in terms of the components of

\footnotetext{
${ }^{5}$ They are no longer AdS scalars if $J \neq 0$ because the twisting happens in the four-dimensional subspace transverse to the AdS momentum, but the polarization can have components along both the AdS momentum and its transverse space.
} 
the conformal algebra, $P_{\mu}, K^{\mu}, D$ and $M_{\mu \nu}$. For the operator $\mathcal{O}_{i}\left(x_{i}^{\mu}\right)$ in the three-point function the conserved charges include

$$
\begin{aligned}
\left\langle P_{i}^{\mu}\right\rangle & =-2 i \sum_{\substack{i \neq j \neq k \neq i \\
\alpha_{k}}} \frac{x_{i j}^{\mu}}{x_{i j}^{2}}, \\
\left\langle D_{i}\right\rangle & =i\left(\Delta_{i}-2 x_{\substack{i \mu j \\
i \neq j \neq k \neq i}} \alpha_{k} \frac{x_{i j}^{\mu}}{x_{i j}^{2}}\right),
\end{aligned}
$$

where $x_{i j}^{\mu}=x_{i}^{\mu}-x_{j}^{\mu}$. The sums are over the other two operators in the three-point function. One can also construct the quadratic Casimir operator

$$
-2\left\langle P_{\mu}\right\rangle\left\langle K^{\mu}\right\rangle+\langle D\rangle^{2}-\frac{1}{2}\left\langle M_{\mu \nu}\right\rangle\left\langle M^{\mu \nu}\right\rangle=-\Delta^{2} .
$$

At the point where the three trajectories intersect these constants of the motion are conserved. It is convenient to shift the operator positions such that the intersection point is at $x^{\mu}=0$. In this case all $\left\langle M_{\mu \nu}\right\rangle=0$ and the conserved charges $\left\langle K_{i}^{\mu}\right\rangle$ satisfy

$$
\left\langle K_{i}^{\mu}\right\rangle=-\frac{\alpha_{1} \alpha_{2} \alpha_{3} \Sigma}{F^{2}} x_{12}^{2} x_{23}^{2} x_{31}^{2}\left\langle P_{i}^{\mu}\right\rangle,
$$

where

$$
F=\alpha_{1} \alpha_{2} x_{12}^{2}+\alpha_{2} \alpha_{3} x_{23}^{2}+\alpha_{3} \alpha_{1} x_{13}^{2} .
$$

It is also convenient to write everything in a manifest $\mathrm{SO}(2, d)$ formalism, with

$$
M_{-1 \mu} \equiv \frac{1}{\sqrt{2}}\left(\kappa P_{\mu}-\kappa^{-1} K_{\mu}\right) \quad M_{d \mu} \equiv \frac{1}{\sqrt{2}}\left(\kappa P_{\mu}+\kappa^{-1} K_{\mu}\right) \quad M_{-1 d} \equiv-D,
$$

where $\kappa$ is arbitrary. In this convention the Casimir operator becomes $-\frac{1}{2}\left\langle M_{r s}\right\rangle\left\langle M^{r s}\right\rangle=$ $-\Delta^{2}, r, s=-1 \ldots d$. Setting $d=4$, we can also combine this with the $\operatorname{SO}(6) R$-symmetry Casimir to write

$$
-\frac{1}{2}\left\langle M_{r s}\right\rangle\left\langle M^{r s}\right\rangle+\frac{1}{2}\left\langle R_{I J}\right\rangle\left\langle R^{I J}\right\rangle=-\Delta^{2}+J^{2},
$$

where $R_{I J}$ are the $R$-symmetry generators with $I, J=5, \ldots, 10$.

If $\Delta$ and $J$ are large, then the $\mathrm{SO}(2,4) \times \mathrm{SO}(6)$ algebra effectively reduces to a 10 dimensional Poincaré algebra. Assuming that all $\left\langle M_{\mu \nu}\right\rangle=0$, and choosing $\kappa$ to be

$$
\kappa=\frac{\sqrt{\alpha_{1} \alpha_{2} \alpha_{3} \Sigma}}{F}\left|x_{12}\right|\left|x_{23}\right|\left|x_{31}\right|
$$

then the only non-zero components in $(2.9)$ are $\left\langle M_{-1 m}\right\rangle, m=0, \ldots, 4$ for all three operators. Assuming $\Delta^{2} \gg 1$, and choosing a basis where the only non-zero $R$-symmetry components are $\left\langle R_{J, 10}\right\rangle$, we can then identify the full 10-dimensional flat-space momentum as

$$
k^{M}=\left(\left\langle M_{-1 m}\right\rangle,\left\langle R_{J, 10}\right\rangle\right),
$$


which has to satisfy the on-shell condition

$$
k \cdot k=-\Delta^{2}+J^{2}=-4 n \sqrt{\lambda} .
$$

If we fill out the algebra to the full superconformal $\operatorname{PSU}(2,2 \mid 4)$, then we also have fermionic generators. These include the supergenerators $Q_{\alpha a}$ and $\widetilde{Q}_{\dot{\alpha}}^{a}$, where $\alpha$ and $\dot{\alpha}$ are space-time spinor indices and $a$ is an $\mathrm{SO}(6)$ spinor index (raised or lowered depending on which spinor representation), and the superconformal generators $S_{\alpha}^{a}$ and $\widetilde{S}_{\dot{\alpha} a}$. We can put these into a form that is manifestly $\mathrm{SU}(2,2) \simeq \mathrm{SO}(2,4)$ covariant by defining

$$
\begin{aligned}
Q_{\dot{a} a}^{1} & \equiv\left(\kappa^{1 / 2} Q_{\alpha a}, \kappa^{-1 / 2} \tilde{S}_{\dot{\alpha} a}\right) \\
Q^{2, \dot{a} a} & \equiv\left(\kappa^{-1 / 2} \varepsilon^{\alpha \beta} S_{\beta}^{a}, \kappa^{1 / 2} \varepsilon^{\dot{\alpha} \dot{\beta}} \tilde{Q}_{\dot{\beta}}^{a}\right)
\end{aligned}
$$

where the lowered $\dot{a}$ is an $\mathrm{SO}(2,4)$ spinor index and the raised $\dot{a}$ is an index for the other spinor representation. The $\kappa$ is the same that appears in (2.9). If we then define two sets of supercharges

$$
Q_{A}^{\mathrm{L}}=Q_{\dot{a} a}^{1}+\gamma_{\dot{b} \dot{a}}^{-1} \gamma_{b a}^{6} Q^{2, \dot{b} b}, \quad Q_{A}^{\mathrm{R}}=-i\left(Q_{\dot{a} a}^{1}-\gamma_{\dot{b} \dot{a}}^{-1} \gamma_{b a}^{6} Q^{2, \dot{b} b}\right),
$$

then in the flat-space limit these approach the usual 10-dimensional super-Poincaré generators with

$$
\left\{Q_{A}^{\mathrm{L}, \mathrm{R}}, Q_{B}^{\mathrm{L}, \mathrm{R}}\right\}=-2\left(\mathrm{P}_{+} \Gamma^{M} C\right)_{A B} P_{M}, \quad P_{M}=\left(M_{-1, m}, R_{J, 10}\right), \quad\left\{Q_{A}^{\mathrm{L}}, Q_{B}^{\mathrm{R}}\right\}=0
$$

for $\Delta \gg 1, J \gg 1$, where $\mathrm{P}_{+}$is the positive-chirality projector. ${ }^{6}$

For a primary operator we have that $\left[S_{\alpha}^{a}, \mathcal{O}(0)\right]=\left[\widetilde{S}_{\dot{\alpha} a}, \mathcal{O}(0)\right]=0$. If we compare this to the new generators in $(2.15)$ we see that this corresponds to

$$
Q_{A}^{\mathrm{L}}= \pm i Q_{A}^{\mathrm{R}}
$$

for the primary operators, where the sign depends on the spinor component. With the operator at $x_{i}^{\mu}=0$ it is clear that the operator's charges have $\left\langle K^{\mu}\right\rangle=\left\langle M_{\mu \nu}\right\rangle=0$. If we then assume that the intersection point is arbitrarily far away, or equivalently we are on a part of the trajectory arbitrarily close to the boundary, then for finite $\kappa$ we have $M_{-1 \mu}=M_{4 \mu}=0, M_{-14}=-i \Delta$, which puts the space-time directions transverse to the momentum in $\mathrm{AdS}_{5}$. Going back to (2.15), we see that in the flat-space limit this imposes the condition on the operators

$$
Q_{\alpha \tilde{a}}^{\mathrm{L}}=i Q_{\alpha \tilde{a}}^{\mathrm{R}}, \quad Q_{\dot{\alpha}}^{\mathrm{L}} \tilde{a}=-i Q_{\dot{\alpha}}^{\mathrm{R}}{ }^{\tilde{a}},
$$

where we use here the explicit four-dimensional space-time $(\alpha, \dot{\alpha})$ and orthogonal six-dimensional ( $\tilde{a})$ spinor indices. Shifting the intersection point back to $x^{\mu}=0$, the $M_{-1 \mu}$ are no longer zero, but the primary operator condition is still defined by the directions transverse

\footnotetext{
${ }^{6}$ For $J \sim \mathcal{O}(1)$, the correction terms in $(2.16)$ are of the same order as the $P_{J}$ components. However, as long as $\Delta \gg 1$ these are suppressed compared to the other momentum components and hence do not affect the conditions obeyed by the vertex operators.
} 
to the momentum in $\mathrm{AdS}_{5}$. Hence, the four-dimensional spinor indices in (2.18) are replaced with the spinor indices for the four-dimensional space transverse to the particle momentum in $\mathrm{AdS}_{5}$, while the six-dimensional spinor indices are replaced accordingly.

We then proceed by finding operators that satisfy the condition in (2.18). As was emphasized in [21], this leads to string states that are linear combinations of NS-NS and R-R modes. At the massless level this gives chiral primary operators while at the massive levels the states correspond only to primary operators. The relative sign in (2.18) complicates matters as the states cannot be scalars in the full 10-dimensional flat-space. At best they can be scalars in the two five-dimensional subspaces. Nonetheless, it is still convenient to start with twisted versions of our vertex operators that correspond to 10-dimensional scalars, and then untwist them afterwards. In particular, if we start with a vertex operator $V_{\mathrm{T}, k}$ that satisfies

$$
\left[Q_{A}^{\mathrm{L}}, V_{\mathrm{T}, k}\right]=\left[i Q_{A}^{\mathrm{R}}, V_{\mathrm{T}, k}\right]
$$

then this is related to an operator $V_{k}$ that satisfies (2.18) by

$$
V_{\mathrm{T}, k}=e^{\pi i\left(M_{0^{\prime} 1^{\prime}}^{\mathrm{R}}+M_{2^{\prime} 3^{\prime}}^{\mathrm{R}}\right)} V_{k}
$$

where the $\mu^{\prime}$ components are transverse to $k^{M}$ in the $\mathrm{AdS}_{5}$ part and $M_{\mu^{\prime} \nu^{\prime}}^{\mathrm{R}}$ is a generator of rotations for the right-movers.

For the level-zero vertex operators, those corresponding to the massless states, a general NS-NS vertex operator in the $(-1,-1)$ picture has the form [33]

$$
W_{1, k}^{(-1,-1)}(z, \bar{z})=g_{\mathrm{c}} \varepsilon_{M \tilde{M}} \psi^{M} e^{-\phi} \tilde{\psi}^{\tilde{M}} e^{-\tilde{\phi}} e^{i k \cdot X}, \quad k^{M} \varepsilon_{M \tilde{M}}=k^{\tilde{M}} \varepsilon_{M \tilde{M}}=0,
$$

where $\phi$ and $\tilde{\phi}$ are the left and right superconformal ghost fields. Eventually we will replace $g_{\mathrm{c}}$ and $\alpha^{\prime}$ with their dictionary values, but for now leave them in the usual string-theory form. Since the total ghost number for the three-point function must be $(-2,-2)$, we will also need the vertex operator in the $(0,0)$ picture, which is given by

$$
W_{1, k}^{(0,0)}(z, \bar{z})=-g_{\mathrm{c}} \varepsilon_{M \tilde{M}} \frac{2}{\alpha^{\prime}}\left(i \partial X^{M}+\frac{\alpha^{\prime}}{2} k \cdot \psi \psi^{M}\right)\left(i \bar{\partial} X^{\tilde{M}}+\frac{\alpha^{\prime}}{2} k \cdot \tilde{\psi} \tilde{\psi}^{\tilde{M}}\right) e^{i k \cdot X} .
$$

A generic vertex operator in the R-R sector is [33]

$$
W_{2, k}^{(-1 / 2,-1 / 2)}(z, \bar{z})=g_{\mathrm{c}} t_{A B} \tilde{\Theta}^{A} e^{-\frac{1}{2} \tilde{\phi}} \Theta^{B} e^{-\frac{1}{2} \phi} e^{i k \cdot X}, \quad t \not k=0,
$$

where $\Theta_{A}$ and $\tilde{\Theta}_{B}$ are the sixteen-component left and right twist fields. We only need these vertex operators in the $(-1 / 2,-1 / 2)$ picture.

The twisted vertex operator will be a linear combination of the NS-NS and the R-R vertex operators. Equation (2.19) can only be satisfied up to spurious terms and has one scalar solution,

$$
W_{\mathrm{T}}=W_{1, \mathrm{~T}}+\frac{1}{\sqrt{2}} W_{2, \mathrm{~T}},
$$


up to a normalization factor. Here, $W_{1, \mathrm{~T}}$ and $W_{2, \mathrm{~T}}$ are the NS-NS and R-R vertex operators with polarizations ${ }^{7}$

$$
\varepsilon_{\mathrm{T}}^{M \tilde{M}}=\eta^{M \tilde{M}}-\frac{k^{M} \bar{k}^{\tilde{M}}+k^{\tilde{M}} \bar{k}^{M}}{k \cdot \bar{k}}, \quad t_{\mathrm{T}, A B}=\left(\frac{\alpha^{\prime}}{2}\right)^{1 / 2}\left(C^{\dagger} \not k\right)_{A B},
$$

where $C$ is the charge conjugation matrix and $\bar{k}^{N}$ is an arbitrary light-like vector satisfying $k \cdot \bar{k} \neq 0$. Untwisting the vertex operators the NS-NS polarization becomes

$$
\varepsilon^{M \tilde{M}}=(-1)^{\sigma_{k}(\tilde{M})} \varepsilon_{\mathrm{T}}^{M \tilde{M}}
$$

where

$$
\sigma_{k}(N)= \begin{cases}1 & N=0^{\prime}, \ldots, 3^{\prime} \\ 0 & N=4^{\prime}, 5, \ldots, 9 .\end{cases}
$$

Untwisting the R-R vertex operator, $t_{\mathrm{T}, A B}$ gets replaced with

$$
t_{A B}=\left(\frac{\alpha^{\prime}}{2}\right)^{1 / 2}\left(C^{\dagger} i \Gamma^{0^{\prime}} \Gamma^{1^{\prime}} \Gamma^{2^{\prime}} \Gamma^{3^{\prime}} \not k\right)_{A B} .
$$

As before, the primed indices denote a frame in which the directions $0^{\prime}, \ldots, 3^{\prime}$ are transverse to $k$. The NS-NS vertex operator is for a graviton component in the full 10-dimensional space-time while the R-R vertex operator is that for a self-dual 4-form field. Further details on the massless vertices can be found in appendix B.1.

Moving now to level one there are two different types of NS-NS vertex operators that we can construct. The first has the form in the $(-1,-1)$ picture [34]

$$
V_{1, k}^{(-1,-1)}(z, \bar{z})=g_{\mathrm{c}} \frac{2}{\alpha^{\prime}} \varepsilon_{M N ; \tilde{M} \tilde{N}} \psi^{M} i \partial X^{N} e^{-\phi} \tilde{\psi}^{\tilde{M}} i \bar{\partial} X^{\tilde{N}} e^{-\tilde{\phi}} e^{i k \cdot X},
$$

where the first two and last two indices of $\varepsilon_{M N ; \tilde{M} \tilde{N}}$ are symmetric and traceless. The second vertex in the $(-1,-1)$ picture is given by

$$
V_{2, k}^{(-1,-1)}(z, \bar{z})=g_{\mathrm{c}} \alpha_{M N L ; \tilde{M} \tilde{N} \tilde{L}} \psi^{M} \psi^{N} \psi^{L} e^{-\phi} \tilde{\psi}^{\tilde{M}} \tilde{\psi}^{\tilde{N}} \tilde{\psi}^{\tilde{L}} e^{-\tilde{\phi}} e^{i k \cdot X} .
$$

where the first three and last three indices of $\alpha_{M N L ; \tilde{M} \tilde{N} \tilde{L}}$ are antisymmetric. For physical states the contraction of any index in $\varepsilon_{M N ; \tilde{M} \tilde{N}}$ or $\alpha_{M N L ; \tilde{M} \tilde{N} \tilde{L}}$ with $k^{M}$ vanishes. There are $44 \times 44$ independent polarizations for $V_{1}$ and $84 \times 84$ for $V_{2}$, but only one of each type is a ten-dimensional Lorentz scalar. These scalars correspond to the twisted vertex operators, $V_{1, \mathrm{~T}}$ and $V_{2, \mathrm{~T}}$. For $V_{1, \mathrm{~T}}$ the unnormalized polarization tensor is given by

$$
\varepsilon_{\mathrm{T}}^{M N ; \tilde{M} \tilde{N}}=\frac{1}{2}\left(\hat{\eta}^{M \tilde{M}} \hat{\eta}^{N \tilde{N}}+\hat{\eta}^{M \tilde{N}} \hat{\eta}^{N \tilde{M}}\right)-\frac{1}{9} \hat{\eta}^{M N} \hat{\eta}^{\tilde{M} \tilde{N}},
$$

where $\hat{\eta}^{M N} \equiv \eta^{M N}-\frac{k^{M} k^{N}}{k^{2}}$. For $V_{2, \mathrm{~T}}$ the unnormalized polarization is

$$
\alpha_{\mathrm{T}}^{M N L ; \tilde{M} \tilde{N} \tilde{L}}=\frac{1}{3 !^{2}}\left(\hat{\eta}^{M \tilde{M}^{2}} \hat{\eta}^{N \tilde{N}} \hat{\eta}^{L \tilde{L}} \mp(5 \text { permutations })\right) .
$$

\footnotetext{
${ }^{7}$ The coefficient of the R-R polarization $t_{A B}$ depends on the chiralities of the spin fields $\tilde{\Theta}^{A}, \Theta^{B}$. Since the worldsheet theory is chiral, we will assume throughout the paper that all spin fields $\Theta, \tilde{\Theta}$ have positive chirality.
} 
We will also need the vertex operators in the $(0,0)$ picture, and these are given by

$$
\begin{aligned}
V_{1, \mathrm{~T}, k}^{(0,0)}= & -g_{\mathrm{c}}\left(\frac{2}{\alpha^{\prime}}\right)^{2} \varepsilon_{\mathrm{T}, M N ; \tilde{M} \tilde{N}}\left(i \partial X^{M}\left(i \partial X^{N}+\frac{\alpha^{\prime}}{2} k \cdot \psi \psi^{N}\right)+\frac{\alpha^{\prime}}{2} \partial \psi^{M} \psi^{N}\right) \\
& \times\left(i \bar{\partial} X^{\tilde{M}}\left(i \bar{\partial} X^{\tilde{N}}+\frac{\alpha^{\prime}}{2} k \cdot \tilde{\psi} \tilde{\psi}^{\tilde{N}}\right)+\frac{\alpha^{\prime}}{2} \bar{\partial} \tilde{\psi}^{\tilde{M}} \tilde{\psi}^{\tilde{N}}\right) e^{i k \cdot X} \\
V_{2, \mathrm{~T}, k}^{(0,0)}= & -g_{\mathrm{c}} \frac{2}{\alpha^{\prime}} \alpha_{\mathrm{T}, M N L ; \tilde{M} \tilde{N} \tilde{L}}\left(3 i \partial X^{M}+\frac{\alpha^{\prime}}{2} k \cdot \psi \psi^{M}\right) \psi^{N} \psi^{L} \\
& \times\left(3 i \bar{\partial} X^{\tilde{M}}+\frac{\alpha^{\prime}}{2} k \cdot \tilde{\psi} \tilde{\psi}^{\tilde{M}}\right) \tilde{\psi}^{\tilde{N}} \tilde{\psi}^{\tilde{L}} e^{i k \cdot X} .
\end{aligned}
$$

The vertex operators for level-one $\mathrm{R}-\mathrm{R}$ string states in the $(-1 / 2,-1 / 2)$ picture were shown to have the form [34]

$$
\begin{aligned}
V_{3, k}^{(-1 / 2,-1 / 2)}(z, \bar{z})= & g_{\mathrm{c}}\left(i \bar{\partial} X^{M} \tilde{\Theta}-\frac{1}{8} \frac{\alpha^{\prime}}{2} \tilde{\psi}^{M}(\not k \tilde{\psi} \tilde{\Theta})\right)^{A} e^{-\tilde{\phi} / 2} \\
& \times t_{M A ; N B}\left(i \partial X^{N} \Theta-\frac{1}{8} \frac{\alpha^{\prime}}{2} \psi^{N}(\not k \psi \Theta)\right)^{B} e^{-\phi / 2} e^{i k X},
\end{aligned}
$$

with the twisted (scalar) states given by $[21]^{8}$

$$
t_{\mathrm{T}, M A ; N B}=\left(\frac{\alpha^{\prime}}{2}\right)^{1 / 2}\left(C^{\dagger} \not k\left(\hat{\eta}_{M N}-\frac{1}{9} \hat{\Gamma}_{M} \hat{\Gamma}_{N}\right)\right)_{A B}, \quad \hat{\Gamma}^{M}=\Gamma^{M}-\not k k^{M} / k^{2} .
$$

If we now take a linear combination of $V_{1, \mathrm{~T}}, V_{2, \mathrm{~T}}$ and $V_{3, \mathrm{~T}}$ and solve for (2.19) up to spurious terms, we find that

$$
V_{\mathrm{T}}=V_{1, \mathrm{~T}}+V_{2, \mathrm{~T}}+\frac{1}{\sqrt{2}} V_{3, \mathrm{~T}}
$$

does the trick up to an overall normalization constant. This vertex can then be untwisted in a fashion similar to the massless case. Further technical details including a careful derivation of the supersymmetry transformations and the normalization factors can be found in appendix B.2.

\section{Three-point functions}

In this section we compute the three-point functions for any combination of chiral primaries and level-one primaries, where we use the properly normalized and untwisted versions of the vertex operators $(2.24),(2.37)$.

The chiral primaries are operators of the form

$$
\mathcal{O}_{\mathrm{CP}}=C_{I_{1} I_{2} \ldots I_{J}} \operatorname{Tr}\left[\Phi^{I_{1}} \Phi^{I_{2}} \ldots \Phi^{I_{J}}\right]
$$

where $C_{I_{1} I_{2} \ldots I_{J}}$ is symmetric and traceless. These are states in the $[0, J, 0]$ representation of $\mathrm{SO}(6)$ and have the protected dimension $\Delta_{\mathrm{CP}}=\Delta_{0}=J$.

\footnotetext{
${ }^{8}$ Again we assume that all spin fields $\Theta, \tilde{\Theta}$ in (2.35) have positive chirality.
} 
The other operators that make up the three-point functions are assumed to be primaries dual to string states at level one. These operators also transform in the $[0, J, 0]$ representation, but now the bare dimension is $\Delta_{0}=J+2$ [35]. Operators of this type include those with the form

$$
\mathcal{O}_{J}=\operatorname{Tr}\left[\Phi^{I} \Phi_{I} Z^{J}\right]+\ldots
$$

where $\Phi_{I}$ are the six scalars of $\mathcal{N}=4 \mathrm{SYM}, Z=\left(\Phi_{5}+i \Phi_{6}\right) / \sqrt{2}$, and the ellipsis refers to different positions of the $\Phi_{I}$ in the trace, such that the corresponding magnon momenta are at level one [35, 36]. If $J>0$ then levels higher than one are possible, but level one is the only possibility for the Konishi operator

$$
\mathcal{O}_{\mathrm{K}}=\mathcal{O}_{J=0}=\operatorname{Tr}\left[\Phi^{I} \Phi_{I}\right]
$$

Our results are applicable if $\Delta \gg 1$. For the chiral primaries this means that $J \gg 1$, but for the level-one primaries small values of $J$ are possible, as long as the $R$-charges in the full three-point function are conserved.

One should note that correlators with two or more twisted operators must vanish due to a supersymmetric Ward identity. ${ }^{9}$ The twisted vertices are annihilated by the sixteen supercharges $Q^{A}=Q_{\mathrm{L}}^{A}-i Q_{\mathrm{R}}^{A}$ and, since they have non-zero momenta, they can be written as the action of those supercharges on another vertex operator $V_{\mathrm{T}}=\left\{Q^{A}, X\right\}$. It is then straightforward to see that

$$
\left\langle V_{1, \mathrm{~T}} V_{2, \mathrm{~T}} V_{3, \mathrm{~T}}\right\rangle=\left\langle X\left[Q^{A}, V_{2, \mathrm{~T}}\right] V_{3, \mathrm{~T}}\right\rangle+\left\langle X V_{2, \mathrm{~T}}\left[Q^{A}, V_{3, \mathrm{~T}}\right]\right\rangle=0 .
$$

If instead we consider the correlator $\left\langle V_{1, \mathrm{~T}} V_{2, \mathrm{~T}} V_{3}\right\rangle$, the same argument follows except that we must choose the supersymmetries that also annihilate the untwisted vertex $V_{3}$, which correspond to the eight supercharges $Q_{\alpha \tilde{a}}^{-}=Q_{\alpha \tilde{a}}^{\mathrm{L}}-i Q_{\alpha \tilde{a}}^{\mathrm{R}}$. In the case $\left\langle V_{1, \mathrm{~T}} V_{2} V_{3}\right\rangle$ of two untwisted vertices with momenta $k_{2,3}$, if one of them is closed under $Q_{\alpha \tilde{a}}^{-}, Q_{\dot{\alpha}}^{+, \tilde{a}}=Q_{\dot{\alpha}}^{\mathrm{L}, \tilde{a}}+$ $i Q_{\dot{\alpha}}^{\mathrm{R}, \tilde{a}}$, then the other is closed under another set of $Q^{ \pm}$, which is obtained from $Q_{\alpha \tilde{a}}^{-}, Q_{\dot{\alpha}}^{+, \tilde{a}}$ by a rotation that takes $k_{2}$ into $k_{3}$. The rotation mixes $\alpha$ with $\dot{\alpha}$ components and lower with upper $\tilde{a}$ components, and thus there is no common supercharge that annihilates both untwisted vertex operators. Hence, only correlators with at least two untwisted vertices can be non-zero.

\subsection{Three chiral primaries}

In this subsection we compute the three-point function for three chiral primaries, showing that it is consistent with the result in [28].

As shown in appendix B.1, the normalized vertex operator satisfying the primary operator condition is given by

$$
W_{k}=-\frac{1}{4}\left(W_{1, k}+\frac{1}{\sqrt{2}} W_{2, k}\right) .
$$

\footnotetext{
${ }^{9}$ We thank E. Witten for a discussion on this point.
} 
We have included an extra sign that is not determined by the $Q_{\mathrm{L}}= \pm i Q_{\mathrm{R}}$ conditions nor by normalization, but is necessary to be consistent with [28]. We will continue to use this choice of sign in the later subsections.

The three-point amplitudes are of two general types, with either zero or two R-R vertex operators inside the amplitude. For NS-NS vertex operators only, two of the vertex operators should be given in the $(-1,-1)$ picture, while one should be in the $(0,0)$ picture to have the correct ghost charge. In appendix C.2 it is shown that

$$
\left\langle W_{1, k_{1}}^{(-1,-1)} W_{1, k_{2}}^{(-1,-1)} W_{1, k_{3}}^{(0,0)}\right\rangle=\frac{4 g_{\mathrm{c}}^{3} \alpha^{\prime} \tilde{\alpha}_{1} \tilde{\alpha}_{2} \tilde{\alpha}_{3} \tilde{\Sigma}}{J_{1}^{2} J_{2}^{2} J_{3}^{2}}\left(J_{1}^{4}+6 J_{1}^{2} J_{2}^{2}+J_{2}^{4}+6 J_{1}^{2} J_{3}^{2}+6 J_{2}^{2} J_{3}^{2}+J_{3}^{4}\right) .
$$

For two R-R vertex operators and one NS-NS, we can choose the R-R vertex operators to be in the $(-1 / 2,-1 / 2)$ picture and the NS-NS in the $(-1,-1)$ picture. In appendix C.2 we then show that

$$
\left\langle W_{1, k_{1}}^{(-1,-1)} W_{2, k_{2}}^{(-1 / 2,-1 / 2)} W_{2, k_{3}}^{(-1 / 2,-1 / 2)}\right\rangle=\frac{32 g_{\mathrm{c}}^{3} \alpha^{\prime} \tilde{\alpha}_{1} \tilde{\alpha}_{2} \tilde{\alpha}_{3} \tilde{\Sigma}}{J_{1}^{2} J_{2} J_{3}}\left(3 J_{1}^{2}+J_{2}^{2}+J_{3}^{2}\right) .
$$

Taking the combination in (3.5) and using (3.6) and (3.7), we find

$$
\left\langle W_{k_{1}} W_{k_{2}} W_{k_{3}}\right\rangle=g_{\mathrm{c}}^{3} \alpha^{\prime} \frac{\tilde{\alpha}_{1} \tilde{\alpha}_{2} \tilde{\alpha}_{3} \tilde{\Sigma}^{5}}{J_{1}^{2} J_{2}^{2} J_{3}^{2}},
$$

with $\tilde{\Sigma}$ and $\tilde{\alpha}_{i}$ defined in $(2.2)$.

Using (1.3), (1.5), (2.3), (2.4) and the AdS/CFT dictionary value $g_{\mathrm{c}}=\pi^{3 / 2} / N$, we obtain

$$
\mathcal{C}_{123} \approx \frac{1}{N} \sqrt{J_{1} J_{2} J_{3}}\left\langle C^{J_{1}} C^{J_{2}} C^{J_{3}}\right\rangle
$$

reproducing the result in [28].

\subsection{Two chiral primaries}

We will next compute the correlator for two chiral primaries and one massive operator $\left\langle W_{k_{1}} W_{k_{2}} V_{k_{3}}\right\rangle$. As shown in appendix B, the normalized vertex operators which satisfy the primary condition are (3.5) and

$$
V_{k}=-\frac{1}{16}\left(V_{1, k}+V_{2, k}+\frac{1}{\sqrt{2}} V_{3, k}\right) .
$$

Here the overall minus sign is again not determined with the primary operator condition, but we make this choice to keep the sign of the structure constants obtained consistent with the ones at weak coupling. Again, we have two types of three-point amplitudes. The first, with no R-R vertex operators, consists of $\left\langle W_{1, k_{1}} W_{1, k_{2}} V_{1, k_{3}}\right\rangle$ and $\left\langle W_{1, k_{1}} W_{1, k_{2}} V_{2, k_{3}}\right\rangle$, where two operators have ghost charges $(-1,-1)$ and one has $(0,0)$. The second group, with two R-R vertices, is formed by $\left\langle W_{2, k_{1}} W_{2, k_{2}} V_{1, k_{3}}\right\rangle,\left\langle W_{2, k_{1}} W_{2, k_{2}} V_{2, k_{3}}\right\rangle$ and $\left\langle W_{1, k_{1}} W_{2, k_{2}} V_{3, k_{3}}\right\rangle$, with the R-R operators in the $(-1 / 2-1 / 2)$ frame and the NS-NS in the $(-1,-1)$. 
All these correlators are computed in appendix C.3. Using (3.5) and (3.10) we find

$$
\left\langle W_{k_{1}} W_{k_{2}} V_{k_{3}}\right\rangle=g_{\mathrm{c}}^{3} \alpha^{\prime 2} \frac{\alpha_{1}^{2} \alpha_{2}^{2} \Sigma^{4} \tilde{\alpha}_{3}^{2} \tilde{\Sigma}^{2}}{\Delta_{1}^{2} \Delta_{2}^{2} \Delta_{3}^{4}} .
$$

For the massless states (3.5), we have $\Delta=J$. When $J_{1}=J_{2} \equiv J$ and $J_{3}=0$, i.e. $\Delta \equiv \Delta_{3}=2 / \sqrt{\alpha^{\prime}}$, the correlator is given by

$$
\left\langle W_{k_{1}} W_{k_{2}} V_{k_{3}}\right\rangle=\frac{g_{\mathrm{c}}^{3}}{16} \alpha^{\prime 2}\left(J+\frac{1}{2} \Delta\right)^{4} .
$$

Using (1.3) and (1.5), the structure constant becomes

$$
\mathcal{C}_{123} \approx \frac{\sqrt{\pi}}{4 N \sqrt{\lambda}} 2^{-\Delta} J^{2(1-J)}\left(J-\frac{1}{2} \Delta\right)^{J-\Delta / 2-1 / 2}\left(J+\frac{1}{2} \Delta\right)^{J+\Delta / 2+3 / 2},
$$

where we used that $\left\langle\psi_{J_{1}} \psi_{J_{2}} \psi_{J_{3}}\right\rangle=1 / \pi^{3 / 2}$ in this case.

We should say that the procedure we are using here requires that the operator dimensions satisfy a triangle rule, $\Delta_{1}+\Delta_{2} \geq \Delta_{3}$, which means that the result in (3.13) is only valid if $J \geq \Delta / 2$. As we approach the extremal case $\Delta_{1}+\Delta_{2}=\Delta_{3}$, the saddle point approaches the boundary [29], and (1.3) develops a singularity [37]. The same thing can happen for three chiral primaries, but in that case the coupling also goes to zero and the overall structure constant stays finite [37]. That is not the case here as the coupling is non-zero at the extremal value. Furthermore, because of $R$-charge conservation it is not possible to go beyond the extremal case for three chiral primaries, but it is possible when one operator is non-chiral. Lastly, it is known that three-point functions of single-trace operators get enhanced at the extremal point due to $1 / N$ mixing with double-trace operators [37]. This all suggests a breakdown of the particle approximation at the extremal point.

\subsection{One chiral primary}

For the computation of the correlator $\left\langle V_{k_{1}} V_{k_{2}} W_{k_{3}}\right\rangle$ we use again the normalizations (3.5) and (3.10). The amplitudes with no R-R vertex operators are $\left\langle V_{1, k_{1}} V_{1, k_{2}} W_{1, k_{3}}\right\rangle$, $\left\langle V_{2, k_{1}} V_{2, k_{2}} W_{1, k_{3}}\right\rangle$ and $\left\langle V_{2, k_{1}} V_{1, k_{2}} W_{1, k_{3}}\right\rangle$, where two operators have ghost charges $(-1,-1)$ and one has $(0,0)$. The second group, with two $\mathrm{R}-\mathrm{R}$ vertices in the $(-1 / 2,-1 / 2)$ picture and the NS-NS vertex in the $(-1,-1)$ picture, is formed by $\left\langle V_{1, k_{1}} V_{3, k_{2}} W_{2, k_{3}}\right\rangle,\left\langle V_{2, k_{1}} V_{3, k_{2}} W_{2, k_{3}}\right\rangle$ and $\left\langle V_{3, k_{1}} V_{3, k_{2}} W_{1, k_{3}}\right\rangle$. Using the formulas for these correlators from appendix C.4, we get in this case a more complicated result

$$
\left\langle V_{k_{1}} V_{k_{2}} W_{k_{3}}\right\rangle=\frac{g_{\mathrm{c}}^{3} \Sigma^{4}}{\Delta_{1}^{4} \Delta_{2}^{4} \Delta_{3}^{2}}\left(-\frac{1}{2} \Omega_{1}^{2} \alpha_{3}^{4}+\alpha_{3}^{2}\left(3 \Omega_{1}^{2}+2 \Omega_{1} \alpha_{3}+\alpha_{3}^{2}\right) \Omega_{4}-\frac{1}{2} \Omega_{1}\left(\Omega_{1}-4 \alpha_{3}\right) \Omega_{4}^{2}+\Omega_{4}^{3}\right),
$$

where

$$
\Omega_{1}=\alpha_{1}+\alpha_{2}, \quad \Omega_{4}=\alpha^{\prime} \alpha_{1} \alpha_{2} \alpha_{3} \Sigma .
$$

The result simplifies if we assume all $J_{i} \ll \lambda^{1 / 4}$, in which case we find

$$
\left\langle V_{k_{1}} V_{k_{2}} W_{k_{3}}\right\rangle \approx \frac{g_{\mathrm{c}}^{3}}{2}\left(1+2 \sqrt{\alpha^{\prime}} J_{3}+\ldots\right) .
$$


Using (1.3), (1.5) we obtain in this limit that the structure constant is

$$
\mathcal{C}_{123} \approx \frac{\pi^{2}}{N} 2^{-J_{3}}\left(\lambda^{1 / 4}+\frac{3}{2} J_{3}+\mathcal{O}\left(\lambda^{0}\right)\right)\left\langle\psi_{J_{1}} \psi_{J_{2}} \psi_{J_{3}}\right\rangle
$$

Choosing $J_{1}=0$ and $J_{2}=J_{3}=J$, it becomes

$$
\mathcal{C}_{123} \approx \frac{1}{N} \pi^{1 / 2}\left(\lambda^{1 / 4}+\frac{3}{2} J\right) 2^{-J}
$$

\subsection{Zero chiral primaries}

Finally, we compute the three-point function of three Konishi-like operators $\left\langle V_{k_{1}} V_{k_{2}} V_{k_{3}}\right\rangle$, using the vertex (3.10). The amplitudes with no R-R vertex operators are $\left\langle V_{1, k_{1}} V_{1, k_{2}} V_{1, k_{3}}\right\rangle$, $\left\langle V_{2, k_{1}} V_{2, k_{2}} V_{2, k_{3}}\right\rangle,\left\langle V_{2, k_{1}} V_{2, k_{2}} V_{1, k_{3}}\right\rangle$ and $\left\langle V_{1, k_{1}} V_{1, k_{2}} V_{2, k_{3}}\right\rangle$, where two operators have ghost charges $(-1,-1)$ and one has ghost charge $(0,0)$. The correlators with two $\mathrm{R}-\mathrm{R}$ vertices in the $(-1 / 2,-1 / 2)$ picture and one NS-NS vertex in the $(-1,-1)$ picture are $\left\langle V_{1, k_{1}} V_{3, k_{2}} V_{3, k_{3}}\right\rangle$ and $\left\langle V_{2, k_{1}} V_{3, k_{2}} V_{3, k_{3}}\right\rangle$. Once again, the details of the calculation of these amplitudes are given in appendix C.5. Putting all cases together we get

$$
\begin{gathered}
\left\langle V_{k_{1}} V_{k_{2}} V_{k_{3}}\right\rangle=\frac{g_{\mathrm{c}}^{3} \Sigma^{4}}{\Delta_{1}^{4} \Delta_{2}^{4} \Delta_{3}^{4}}\left(\frac{1}{2} \Sigma_{2}^{4}+\frac{9}{2} \Sigma_{4}^{2}+\left(2 \Sigma_{2}^{3}-3 \Sigma^{2} \Sigma_{2}^{2}+6 \Sigma^{2} \Sigma_{4}+3 \Sigma_{2} \Sigma_{4}\right) \alpha^{\prime} \Sigma_{4}\right. \\
\left.+\frac{1}{2}\left(3 \Sigma^{4}+7 \Sigma_{2}^{2}-8 \Sigma^{2} \Sigma_{2}+6 \Sigma_{4}\right)\left(\alpha^{\prime} \Sigma_{4}\right)^{2}-\left(\Sigma^{2}-3 \Sigma_{2}\right)\left(\alpha^{\prime} \Sigma_{4}\right)^{3}+\left(\alpha^{\prime} \Sigma_{4}\right)^{4}\right) .
\end{gathered}
$$

where

$$
\Sigma_{2}=\alpha_{1} \alpha_{2}+\alpha_{1} \alpha_{3}+\alpha_{2} \alpha_{3}, \quad \Sigma_{4}=\alpha_{1} \alpha_{2} \alpha_{3} \Sigma,
$$

If we now consider the case of all $J_{i}=0$ (i.e. $\Delta_{i}=2 / \sqrt{\alpha^{\prime}}$ ), we obtain the three-point function (C.68) for three Konishi operators

$$
\left\langle V_{k_{1}} V_{k_{2}} V_{k_{3}}\right\rangle \approx g_{\mathrm{c}}^{3} \frac{3^{8}}{2^{9}} .
$$

Plugging this result in (1.3), we find the structure constant in (1.8),

$$
\mathcal{C}_{123} \approx \frac{1}{N} 64 \pi^{1 / 2} \lambda^{1 / 4}\left(\frac{3}{4}\right)^{3 \lambda^{1 / 4}+5 / 2}
$$

\section{Discussion}

In this work we computed the three-point functions for certain short scalar operators in $\mathcal{N}=4 \mathrm{SYM}$ at strong coupling. The set of operators we considered are the chiral primaries as well as primaries which are dual to string states at the first massive level, which includes the Konishi operator. Following the analysis in $[21,29,38]$, the action is dominated by a saddle point composed of three geodesic trajectories propagating from the AdS boundary to a common intersection point, whose location is determined by the conservation of the canonical momentum. For short operators (whose dimensions scale as $\Delta \sim \sqrt[4]{\lambda}$ ), the interaction region is small compared to the AdS radius, and hence 
the coupling at the intersection point is well approximated by a flat-space correlator of appropriate type IIB string vertex operators. For primary operators, the vertex operators have to satisfy a twisted version of the $Q_{\mathrm{L}}=i Q_{\mathrm{R}}$ equation. This constraint uniquely singles out the appropriate string states at the massless and first massive level. ${ }^{10}$ The corresponding vertex operators are certain twisted linear combinations of ten-dimensional flat-space NS-NS and R-R scalar modes. In addition, there is a contribution from the wave-function overlap on the $\mathrm{S}^{5}$ part of the space.

Identifying the correct string vertex operators corresponding to given local operators in the boundary CFT is a long-standing open problem in the AdS/CFT duality. The procedure applied here could provide insights as to what the full $\mathrm{AdS}_{5} \times \mathrm{S}^{5}$ vertex operators might look like. It should also be mentioned that the general method used here and in [21] is not just restricted to $\mathcal{N}=4 \mathrm{SYM}$ with its IIB dual in $\mathrm{AdS}_{5} \times \mathrm{S}^{5}$. It should in principle apply to all AdS/CFT theories with a string theory as a gravitational dual, up to the construction of the appropriate vertex operators. Here we have relied on $\mathcal{N}=4$ supersymmetry to identify the vertex operators.

Our results for the structure constants for any combination of scalar massless and level-one states are given in (1.7), (3.9), (3.13), and (3.17) above. Carrying out the contractions of the primary vertices requires substantial effort due to the combinatorial complexity. Moreover, many intermediate results (partial correlators) contain unappealing prime factors. In comparison, the complete three-point correlators are astonishingly simple. Of course this is not completely unexpected, as planar $\mathcal{N}=4 \mathrm{SYM}$ is believed to be integrable. ${ }^{11}$ The simplicity of our results suggests that integrability will play an equally important role in the computation of three-point functions as it did for the spectral problem. But even disregarding integrability, our results demonstrate that the holographic computation of massive correlators, albeit technically involved, are in fact feasible.

We find that the structure constants for massive states typically are exponentially small in the scaling dimension $\Delta \sim \sqrt[4]{\lambda}$. However, the suppression is still moderate in interesting regimes. For example, the structure constant (1.8) for three Konishi states is $\mathcal{C}_{123} \approx 2.4 / N$ for $\lambda=10^{3}$, a regime in which the Konishi dimension clearly shows GKP behavior [2] and hence the semiclassical approximation should be applicable. Also, in the case of one chiral primary and two massive primaries, the suppression is only exponential in the R-charge $J$ of the chiral primary operator.

There are several directions for future work: it would be very interesting to compute subleading corrections to our results in the $\alpha^{\prime}$ expansion. Subleading terms can be obtained by inserting the expansions for the dimensions $\Delta_{1,2,3}$ of the respective operators, and by calculating loop corrections to the worldsheet amplitude of the appropriate untwisted vertex operators. Analytic corrections to our results will be an expansion in powers of $\alpha^{\prime}$. There is a possibility of non-analytic corrections that come with half-integer powers, as happens for the operator dimensions [8]. But we suspect that such terms only enter the correlators indirectly, through the corrections to the operator dimensions. The three-point vertices

\footnotetext{
${ }^{10}$ There is only one long multiplet at the first massive level, and hence a unique solution to $Q_{\mathrm{L}}=i Q_{\mathrm{R}}$.

${ }^{11}$ See [1] for an introductory review.
} 
are functions of the momenta, whose corrections will come in half-integer powers of $\alpha^{\prime}$ through their dependence on the scaling dimensions. It is also likely that (1.3) itself will get modified as we move away from the point-like limit, as indicated by the singularities that appear as we approach the extremal limit.

Another exciting venture would be to study four-point functions of short operators, aiming at a holographic operator product expansion. For related references, see e.g. [39-43]. It can be hoped that the methods used here will also be useful for higher-point correlators. Likewise, information from the four-point correlation functions of protected operators can be used to check the validity of our results. For example, in [43] there is a prediction for the structure constant between two operators dual to the dilaton and a third operator dual to a higher-spin state on the graviton Regge trajectory. It would be interesting to find their result for the spin 4 operator, which is at the first massive level, using the methods here.

Finally, a bigger goal would be to employ integrability in the computation of shortstring correlators. Based on the experience that integrability techniques usually start with a large-volume limit, this would probably require the adaption of a full thermodynamic Bethe ansatz [44-48] or quantum spectral curve [49] machinery.

\section{Acknowledgments}

We thank M. Bianchi, F. Morales, J. Penedones, O. Schlotterer and E. Witten for discussions. This research is supported in part by Vetenskapsrådet under grant \#2012-3269. The work of TB is supported by a Marie Curie International Outgoing Fellowship within the $7^{\text {th }}$ European Community Framework Programme under grant PIOF-GA-2011-299865. JAM thanks the CTP at MIT and the Galileo Galilei Institute for kind hospitality during the course of this work.

\section{A Bosonization setup and OPE's}

We follow the conventions of Polchinski [50]. In all products of operators evaluated at the same worldsheet point, normal ordering is implicitly assumed.

Bosonization. For OPE's and correlators involving R-R vertices, we use bosonization as described in $[33,51]$. Namely, the ten real fermions $\psi^{M}$ are written as five complex bosons $\phi^{j}$. First convert the $\psi^{M}$ to a Cartan-Weyl basis,

$$
\psi^{ \pm e_{0}}=\frac{1}{\sqrt{2}}\left( \pm \psi^{0}+\psi^{1}\right), \quad \psi^{ \pm e_{j}}=\frac{1}{\sqrt{2}}\left(\psi^{2 j} \pm i \psi^{2 j+1}\right), \quad j=1, \ldots, 4 .
$$

Here, $e_{j}$ denotes a unit vector in direction $j$ on the Euclidean weight lattice of $\mathfrak{o}(1,9)$, where $\pm e_{i} \pm e_{j}$ form the non-zero roots. Now write the $\psi^{ \pm e_{j}}$ as

$$
\psi^{ \pm e_{j}}(z)=e^{ \pm e_{j} \cdot \phi(z)} c_{ \pm e_{j}}, \quad c_{ \pm e_{j}}=(-1)^{N_{1}+\ldots+N_{j-1}}=e^{i \pi\left( \pm e_{j} \cdot M \cdot N\right)},
$$

where $\phi=\left(\phi^{1}, \ldots, \phi^{5}\right)$ is the vector of bosonic fields, and $c_{ \pm e_{j}}$ is a "cocycle factor" that ensures the right commutation relations: $N=\left(N_{1}, \ldots, N_{5}\right)$ is the vector of fermion number 
operators $N_{j}$ for the fermions $\psi^{ \pm e_{j}}$, and $M$ is a lower-triangular sign matrix, given in appendix $\mathrm{E}$ of [51]. More generally, any operator of conformal weight $\lambda$ can be written as $e^{\lambda \cdot \phi} c_{\lambda}$. In particular, the twist fields $\Theta^{A}$ become

$$
\Theta^{A}=e^{A \cdot \phi} c_{A}, \quad c_{A}=e^{i \pi A \cdot M \cdot N},
$$

where $A=( \pm, \ldots, \pm) / 2$ is a five-component spinor weight. Now the OPE for two bosonized operators of weight $\lambda, \lambda^{\prime}$ takes the form

$$
\begin{aligned}
e^{\lambda \cdot \phi\left(z_{1}\right)} c_{\lambda} e^{\lambda^{\prime} \cdot \phi\left(z_{2}\right)} c_{\lambda^{\prime}} & =z_{12}^{\lambda \cdot \lambda^{\prime}} e^{i \pi \lambda \cdot M \cdot \lambda^{\prime}}: e^{\lambda \cdot \phi\left(z_{1}\right)+\lambda^{\prime} \cdot \phi\left(z_{2}\right)}: c_{\lambda+\lambda^{\prime}} \\
& =z_{12}^{\lambda \cdot \lambda^{\prime}} e^{i \pi \lambda \cdot M \cdot \lambda^{\prime}}: e^{\left(\lambda+\lambda^{\prime}\right) \cdot \phi\left(z_{2}\right)}\left(1+z_{12} \lambda \cdot \partial \phi\left(z_{2}\right)+\mathcal{O}\left(z_{12}\right)^{2}\right): c_{\lambda+\lambda^{\prime}}
\end{aligned}
$$

Including the bosonic ghost field $\phi_{\mathrm{g}} \equiv \phi^{6}$, the weight lattice becomes Lorentzian with a "timelike" sixth dimension, and with the sign matrix $M$ now given by (E.7) in [51]. The OPE formula (A.4) continues to hold. For example,

$$
\psi^{ \pm e_{j}} e^{-\phi_{\mathrm{g}}}=e^{\lambda \cdot \phi} c_{\lambda}, \quad \lambda= \pm e_{j}+e_{6}, \quad c_{\lambda}=e^{i \pi \lambda \cdot M \cdot N} .
$$

Using the OPE (A.4), the correlator of $n$ fields with (six-dimensional) weights $\lambda_{1}, \ldots, \lambda_{n}$ becomes

$$
\left\langle e^{\lambda_{1} \cdot \phi\left(z_{1}\right)} c_{\lambda_{1}} \ldots e^{\lambda_{n} \cdot \phi\left(z_{n}\right)} c_{\lambda_{n}}\right\rangle=\delta\left(\sum_{j} \lambda_{j}-2 e_{6}\right) \prod_{1 \leq j<k \leq n} z_{j k}^{\lambda_{j} \cdot \lambda_{k}} e^{i \pi \lambda_{j} \cdot M \cdot \lambda_{k}}
$$

where the scalar product in the exponents is Lorentzian with signature $(1, \ldots, 1,-1)$.

Gamma matrices. In the Cartan-Weyl basis, we use the gamma matrices

$$
\left(\Gamma^{ \pm e_{k}}\right)_{B}^{A}=\sqrt{2} e^{i \pi\left( \pm e_{k}\right) \cdot M \cdot A} \delta\left( \pm e_{k}+A, B\right), \quad k=1, \ldots, 5, \quad A=( \pm, \ldots, \pm) / 2 .
$$

They are related to the gamma matrices $\Gamma^{M}$ in the covariant basis in the same way $\psi^{ \pm e_{j}}$ are related to $\psi^{M}$ (A.1). For the charge conjugation matrix we use

$$
\begin{aligned}
C^{A B} & =e^{i \pi 3 / 4} \sigma^{2} \otimes \sigma^{1} \otimes \sigma^{2} \otimes \sigma^{1} \otimes \sigma^{2} \\
& =\delta(A+B) \begin{cases}e^{-i \pi A_{-1 / 2} \cdot M \cdot A_{-1 / 2}} & \text { for } A \text { with positive chirality } \\
-e^{-i \pi A_{+1 / 2} \cdot M \cdot A_{+1 / 2}} & \text { for } A \text { with negative chirality }\end{cases}
\end{aligned}
$$

Here, $\sigma^{j}$ are the usual Pauli matrices, and $A_{ \pm 1 / 2}=\left(A, \pm \frac{1}{2}\right)$ is the spinor weight $A$ extended by the ghost weight $\pm 1 / 2$.

OPE's. The non-vanishing OPE's among the elementary fields are

$$
X_{i}^{M} X_{j}^{N}=-\frac{\alpha^{\prime}}{2} \eta^{M N} \log \left|z_{i j}\right|^{2}+: X_{i}^{M} X_{j}^{N}:, \quad \psi_{i}^{M} \psi_{j}^{M}=\frac{\eta^{M N}}{z_{i j}}+: \psi_{i}^{M} \psi_{j}^{N}:,
$$

where subscript ${ }_{i}$ denotes dependence on worldsheet coordinates $\left(z_{i}, \bar{z}_{i}\right)$, and $z_{i j} \equiv z_{i}-z_{j}$. In three-point correlators, one often needs to use the OPE of a coordinate derivative with two plane-wave factors, which takes the convenient form

$$
i \partial X_{1}^{M} e^{i k_{2} \cdot X_{2}} e^{i k_{3} \cdot X_{3}}=\frac{\alpha^{\prime}}{2} \frac{k_{2} z_{23}}{z_{12} z_{13}} e^{i k_{2} \cdot X_{2}} e^{i k_{3} \cdot X_{3}}+\ldots \quad \text { for } k_{1}+k_{2}+k_{3}=0 .
$$


For the correlator of plane-wave factors, we use

$$
\left\langle\prod_{j=1}^{n}: e^{i k_{j} \cdot X_{j}}:\right\rangle=\delta\left(\sum_{j} k_{j}\right) \prod_{1 \leq j<k \leq n}\left|z_{j k}\right|^{\alpha^{\prime} k_{j} \cdot k_{k}} .
$$

where for simplicity of notation we write $\delta\left(\sum_{j} k_{j}\right)=(2 \pi)^{10} \delta^{10}\left(\sum_{j} k_{j}\right)$. The OPE's for bosonized fields are given in (A.4). Further relevant OPE's for bosonized fields $\phi^{j}$ are $(j=1, \ldots, 6)$

$$
\begin{aligned}
\phi^{i}\left(z_{1}\right) \phi^{j}\left(z_{2}\right) & =\gamma^{i j} \log \left|z_{12}\right|^{2}+: \phi^{i}\left(z_{1}\right) \phi^{j}\left(z_{2}\right):, \quad \gamma=\operatorname{diag}(1,1,1,1,1,-1), \\
\partial \phi^{j}\left(z_{1}\right) e^{\lambda \cdot \phi\left(z_{2}\right)} & =\frac{\lambda \cdot e_{j}}{z_{12}} e^{\lambda \cdot \phi\left(z_{2}\right)}+: \partial \phi^{j}\left(z_{1}\right) e^{\lambda \cdot \phi\left(z_{2}\right)}:
\end{aligned}
$$

When computing three-point functions, the worldsheet integrations will be replaced by the corresponding $c, \tilde{c}$ ghosts. Three-point correlators are then accompanied by the following expectation value

$$
\left\langle c_{1} c_{2} c_{3} \tilde{c}_{1} \tilde{c}_{2} \tilde{c}_{3}\right\rangle=\left|z_{12}\right|^{2}\left|z_{13}\right|^{2}\left|z_{23}\right|^{2}
$$

For simplicity of notation, in the following sections we will omit these ghosts from the correlators and their presence will be implicit, making the correlators have no dependence on the worldsheet coordinates.

Point-splitting. We define products of fields evaluated at the same worldsheet coordinate by a point-splitting regularization, see e.g. [34]. Specifically,

$$
\begin{aligned}
& \psi^{+e_{i}} \psi^{+e_{i}}(z)=\psi^{-e_{i}} \psi^{-e_{i}}(z)=0, \\
& \psi^{+e_{i}} \psi^{-e_{i}}(z)=-\psi^{-e_{i}} \psi^{+e_{i}}(z)=\partial \phi^{i}(z)
\end{aligned}
$$

Moreover, for $i \neq j$ (the two signs \pm are independent),

$$
\begin{aligned}
& \psi^{ \pm e_{i}} \psi^{ \pm e_{j}} \Theta^{A}(z)=e^{i \pi\left( \pm e_{i}\right) \cdot M \cdot\left( \pm e_{j}\right)} e^{i \pi\left( \pm e_{i} \pm e_{j}\right) \cdot M \cdot A} \\
& \cdot \begin{cases}\left( \pm e_{i} \pm e_{j}\right) \cdot \partial \phi \Theta^{A \pm e_{i} \pm e_{j}}(z) & \text { for }\left( \pm e_{i} \pm e_{j}\right) \cdot A=-1 \\
\Theta^{A \pm e_{i} \pm e_{j}}(z) & \text { for }\left( \pm e_{i} \pm e_{j}\right) \cdot A=0 \\
0 & \text { for }\left( \pm e_{i} \pm e_{j}\right) \cdot A=1\end{cases}
\end{aligned}
$$

Here, the boldface $\Theta^{A}$ is defined as in (A.3), but also for non-spinorial weight $A$. Applying the point-splitting prescription to the term $\psi^{ \pm e_{k}}(\psi \Theta)^{A}$ appearing in the vertex (2.35) yields

$$
\psi^{ \pm e_{k}}(\psi \Theta)^{A}=\delta\left(A_{k} \mp \frac{1}{2}\right) 4 \sqrt{2} e^{ \pm i \pi e_{k} \cdot M \cdot A} \Theta^{A \pm e_{k}}+\left(2 A \pm 6 e_{k}\right) \cdot \partial \phi\left(\Gamma^{ \pm e_{k}} \Theta\right)^{A} .
$$

This also implies

$$
(\psi \psi \Theta)^{A}=36(A \cdot \partial \phi) \Theta^{A} .
$$


When computing correlation functions, terms like (A.17) will always appear multiplied by the polarization tensor $t_{\tilde{M} \tilde{A}, M A}$ which obeys $t_{\tilde{M} \tilde{A}, M A}\left(\Gamma^{M}\right)_{C}^{A}=0$, so we are free to add terms of the form $B \cdot \partial \phi\left(\Gamma^{ \pm e_{k}}\right)_{B}^{A} \Theta^{B}$ to (A.17). We will then use the following formula

$$
\psi^{ \pm e_{k}}(\psi \Theta)^{A} \simeq \delta\left(A_{k} \mp \frac{1}{2}\right) 4 \sqrt{2} e^{ \pm i \pi e_{k} \cdot M \cdot A} \Theta^{A \pm e_{k}}-\frac{8}{5}\left(A \mp \frac{3}{2} e_{k}\right) \cdot \partial \phi\left(\Gamma^{ \pm e_{k}} \Theta\right)^{A},
$$

when computing three-point functions. This will ensure a manifestly correct dependence on the worldsheet coordinates, since $\left(A \mp \frac{3}{2} e_{k}\right) \cdot\left(A \pm e_{k}\right)=0$.

\section{B Primary vertex operators}

To find the massless and massive primary operators, one needs to compute the action of the supersymmetries on the flat-space vertices. We will act with the left supercharge in the $(+1 / 2)$ picture on the NS-NS vertices, and compare to the action of the right supercharge in the $(-1 / 2)$ picture on the $\mathrm{R}-\mathrm{R}$ vertices. The supercharges are given by [34]

$$
Q_{\mathrm{R}}^{A(-1 / 2)}=\oint \frac{d \bar{z}}{2 \pi i} \tilde{\Theta}^{A} e^{-\tilde{\phi}_{6} / 2}
$$

and, using the definition of $Q_{\mathrm{BRST}}$ from [50], we can change to the frame with ghost charge $1 / 2$ in the following way ${ }^{12}$

$$
Q_{\mathrm{L}}^{A(+1 / 2)}=\left[i Q_{\mathrm{BRST}}, \xi Q_{\mathrm{L}}^{A(-1 / 2)}\right]=\frac{1}{\sqrt{2}}\left(\frac{2}{\alpha^{\prime}}\right)^{1 / 2} \oint \frac{d z}{2 \pi i} i \partial X^{M}\left(\Gamma_{M} \Theta\right)^{A} e^{+\phi_{6} / 2} .
$$

\section{B.1 Massless vertex operators}

The supercharges act on the massless vertices in the following way

$$
\left[Q_{\mathrm{L}}^{A(+1 / 2)}, W_{1, \mathrm{~T}}^{(-1,-1)}\right]=i \frac{g_{\mathrm{c}}}{2}\left(\frac{\alpha^{\prime}}{2}\right)^{1 / 2} \varepsilon_{\mathrm{T}, M N}\left(\not k \Gamma^{M}\right)^{A}{ }_{B} \Theta^{B} e^{-\phi_{6} / 2} \tilde{\psi}^{N} e^{-\tilde{\phi}_{6}} e^{i k \cdot X}
$$

and

$$
\left[i Q_{\mathrm{R}}^{A(-1 / 2)}, W_{2, \mathrm{~T}}^{(-1 / 2,-1 / 2)}\right]=-i \frac{g_{\mathrm{c}}}{\sqrt{2}}\left(\frac{\alpha^{\prime}}{2}\right)^{1 / 2}\left(\Gamma_{M} \not k\right)_{B}^{A} \Theta^{B} e^{-\phi_{6} / 2} \tilde{\psi}^{M} e^{-\tilde{\phi}_{6}} e^{i k \cdot X} .
$$

Since $\not k k=0$ and

$$
k_{M} \tilde{\psi}^{M} e^{-\tilde{\phi}_{6}} e^{i k \cdot X}
$$

is a spurious term, then we can write

$$
\left[i Q_{\mathrm{R}}^{A(-1 / 2)}, W_{2, \mathrm{~T}}^{(-1 / 2,-1 / 2)}\right]=i \frac{g_{\mathrm{c}}}{\sqrt{2}}\left(\frac{\alpha^{\prime}}{2}\right)^{1 / 2} \varepsilon_{\mathrm{T}, M N}\left(\not k \Gamma^{M}\right)_{B}^{A} \Theta^{B} e^{-\phi_{6} / 2} \tilde{\psi}^{N} e^{-\tilde{\phi}_{6}} e^{i k \cdot X} .
$$

\footnotetext{
${ }^{12}$ We will need this operator for $A$ with positive chirality. For $A$ with negative chirality, (B.2) would have the opposite sign. Note also that the $i$ in front of $Q_{\mathrm{BRST}}$ is different from the convention in [33], but necessary to get the overall sign of the vertex operators in the $(0,0)$ frame consistent with [50].
} 
We then get that the massless vertex operator must be of the form $W_{\mathrm{T}} \sim W_{1, \mathrm{~T}}+\frac{1}{\sqrt{2}} W_{2, \mathrm{~T}}$. Its normalization is

$$
\frac{1}{g_{\mathrm{c}}^{2}}\left\langle\left(W_{1, \mathrm{~T}}^{k}+\frac{1}{\sqrt{2}} W_{2, \mathrm{~T}}^{k}\right)^{\dagger}\left(W_{1, \mathrm{~T}}^{k}+\frac{1}{\sqrt{2}} W_{2, \mathrm{~T}}^{k}\right)\right\rangle=8+8=16 .
$$

Note that the vertex operator $\left(W_{1, \mathrm{~T}}^{k}+\frac{1}{\sqrt{2}} W_{2, \mathrm{~T}}^{k}\right)^{\dagger}$ satisfies the opposite condition $Q_{\mathrm{L}}^{A}=$ $-i Q_{\mathrm{R}}^{A}$. The normalized massless primary state is then given by

$$
W_{\mathrm{T}}=\frac{1}{4}\left(W_{1, \mathrm{~T}}+\frac{W_{2, \mathrm{~T}}}{\sqrt{2}}\right) .
$$

\section{B.2 Massive vertex operators}

The calculation for the massive case is quite lengthy and tedious so we will present only guidelines of the computation. Starting with $V_{1, \mathrm{~T}}$ and using that $\varepsilon_{\mathrm{T}, M N, \tilde{M} \tilde{N}}$ is symmetric, traceless and orthogonal to $k$, one can obtain

$$
\begin{aligned}
{\left[Q_{\mathrm{L}}^{A(+1 / 2)}, V_{1, \mathrm{~T}}^{(-1,-1)}\right]=} & i \frac{g_{\mathrm{c}}}{2}\left(\frac{2}{\alpha^{\prime}}\right)^{1 / 2}\left(\not k \hat{\Gamma}^{M}\right)_{B}^{A}\left(i \partial X_{N} \Theta-\frac{1}{8} \frac{\alpha^{\prime}}{2} \psi_{N}(\not k \psi \Theta)\right)^{B} e^{-\phi_{6} / 2} \hat{\eta}^{N P} \\
& \times\left(\frac{1}{2} \tilde{\psi}_{\{M} i \bar{\partial} X_{P\}}-\frac{1}{9} \hat{\eta}_{M P}(\tilde{\psi} \cdot i \bar{\partial} X)\right) e^{-\tilde{\phi}_{6}} e^{i k \cdot X}
\end{aligned}
$$

Since we can add total derivatives to the vertices, one can use the following equation to eliminate terms with $\partial \phi_{6}$

$$
\frac{1}{2} \partial \phi_{6} \Theta^{B} e^{-\phi_{6} / 2} e^{i k \cdot X}=i k \cdot \partial X \Theta^{B} e^{-\phi_{6} / 2} e^{i k \cdot X}+\frac{1}{36}(\psi \psi \Theta)^{B} e^{-\phi_{6} / 2} e^{i k \cdot X} .
$$

Using that $\alpha_{\mathrm{T}, M N P, \tilde{M} \tilde{N} \tilde{P}}$ is antisymmetric and noting that

$$
\left(\frac{1}{48}\left(\Gamma_{L}+\frac{6}{k^{2}} \not k k_{L}\right)^{A B} \psi^{L}(\psi \Theta)^{B}+\left(k_{L}-\frac{1}{4} \not k \Gamma_{L}\right)^{A B} i \partial X^{L} \Theta^{B}\right) e^{-\phi_{6} / 2} e^{i k \cdot X}
$$

is a spurious term, then we obtain that the action of $Q_{\mathrm{L}}$ on $V_{2, \mathrm{~T}}$ is

$$
\begin{aligned}
{\left[Q_{\mathrm{L}}^{A(+1 / 2)}, V_{2, \mathrm{~T}}^{(-1,-1)}\right]=} & i \frac{g_{\mathrm{c}}}{4}\left(\frac{2}{\alpha^{\prime}}\right)^{1 / 2}\left(\hat{\Gamma}_{N} \hat{\Gamma}_{P}\left(\hat{\eta}_{M R}-\frac{1}{9} \hat{\Gamma}_{M} \hat{\Gamma}_{R}\right)\right)_{B}^{A} \\
& \times\left(i \partial X^{R} \Theta-\frac{1}{8}\left(\frac{\alpha^{\prime}}{2}\right) \psi^{R}(\not k \psi \Theta)\right)^{B} e^{-\phi_{6} / 2} \tilde{\psi}^{M} \tilde{\psi}^{N} \tilde{\psi}^{P} e^{-\tilde{\phi}_{6}} e^{i k \cdot X}
\end{aligned}
$$

Finally we need only to compute the action of $i Q_{\mathrm{R}}$ on $V_{3, \mathrm{~T}}$. Using that $t_{\mathrm{T}, \tilde{M} A, M B}$ is orthogonal to $k$ and $\left(\Gamma^{M} C t_{\mathrm{T}, M, N}\right)=0$, eliminating terms with $\partial \phi_{6}$ using

$$
\bar{\partial} \tilde{\phi}_{6} \tilde{\psi}^{M} e^{-\tilde{\phi}_{6}} e^{i k \cdot X}=i k \cdot \bar{\partial} X \tilde{\psi}^{M} e^{-\tilde{\phi}_{6}} e^{i k \cdot X}+\bar{\partial} \tilde{\psi}^{M} e^{-\tilde{\phi}_{6}} e^{i k \cdot X},
$$

and noting that the following are spurious terms [34]

$$
\left(\frac{2}{\alpha^{\prime}} \frac{1}{2} i \bar{\partial} X^{[M} \tilde{\psi}^{P]}-\frac{1}{2} \tilde{\psi}^{M} \tilde{\psi}^{R} \tilde{\psi}^{P} k_{R}\right)\left(\hat{\Gamma}_{P} C t_{\mathrm{T}, M A ; N B}\right) e^{-\tilde{\phi}_{6}} e^{i k \cdot X}
$$




$$
\left(\bar{\partial} \tilde{\psi}^{M}+\frac{1}{2} i \bar{\partial} X^{\{M} \tilde{\psi}^{P\}} k_{P}\right)\left(\not k C t_{\mathrm{T}, M A ; N B}\right) e^{-\tilde{\phi}_{6}} e^{i k \cdot X}
$$

we obtain that

$$
\begin{aligned}
& {\left[i Q_{\mathrm{R}}^{A(-1 / 2)}, V_{3, \mathrm{~T}}^{(-1 / 2,-1 / 2)}\right]=} \\
& \left(\left(\frac{i}{2} \bar{\partial} X^{\{M} \tilde{\psi}^{P\}} \hat{\Gamma}_{P}+\frac{1}{4}\left(\frac{\alpha^{\prime}}{2}\right) \tilde{\psi}^{M} \tilde{\psi}^{P} \tilde{\psi}^{R} \hat{\Gamma}_{P} \hat{\Gamma}_{R}\right) \not k\left(\hat{\eta}_{M N}-\frac{1}{9} \hat{\Gamma}_{M} \hat{\Gamma}_{N}\right)\right)_{B}^{A} e^{-\tilde{\phi}_{6}} \\
& \quad \times\left(-i \frac{g_{\mathrm{c}}}{\sqrt{2}}\right)\left(\frac{2}{\alpha^{\prime}}\right)^{1 / 2}\left(i \partial X^{N} \Theta-\frac{1}{8}\left(\frac{\alpha^{\prime}}{2}\right) \psi^{N}(\not k \psi \Theta)\right)^{B} e^{-\phi_{6} / 2} e^{i k \cdot X} .
\end{aligned}
$$

We can now easily see from equations (B.9), (B.12), (B.16) that the primary constraint is satisfied for a vertex operator of the form $V_{\mathrm{T}} \sim V_{1, \mathrm{~T}}+V_{2, \mathrm{~T}}+\frac{1}{\sqrt{2}} V_{3, \mathrm{~T}}$. To normalize it we only need to compute the two-point function with its adjoint, which gives

$$
\frac{1}{g_{\mathrm{c}}^{2}}\left\langle\left(V_{1, \mathrm{~T}}^{k}+V_{2, \mathrm{~T}}^{k}+\frac{1}{\sqrt{2}} V_{3, \mathrm{~T}}^{k}\right)^{\dagger}\left(V_{1, \mathrm{~T}}^{k}+V_{2, \mathrm{~T}}^{k}+\frac{1}{\sqrt{2}} V_{3, \mathrm{~T}}^{k}\right)\right\rangle=44+84+128=256 .
$$

So we have finally obtained that the primary vertex for the first massive level is

$$
V_{\mathrm{T}}=\frac{1}{16}\left(V_{1, \mathrm{~T}}+V_{2, \mathrm{~T}}+\frac{V_{3, \mathrm{~T}}}{\sqrt{2}}\right) .
$$

\section{B.3 Untwisting}

The primary constraint (2.19) was for the twisted vertex operators $W_{\mathrm{T}}$ and $V_{\mathrm{T}}$, but we must compute the three-point function with the untwisted versions of these operators, $W$ and $V$. For the massless vertex the modifications are

$$
\begin{aligned}
\varepsilon_{M, \tilde{M}}^{k_{i}} & =(-1)^{\sigma_{k_{i}}(\tilde{M})} \varepsilon_{\mathrm{T}, M, \tilde{M}}^{k_{i}}, \\
t_{A, B}^{k_{i}} & =\left(\frac{\alpha^{\prime}}{2}\right)^{1 / 2}\left(\mathcal{C}^{\dagger} i \Gamma^{0^{\prime}} \Gamma^{1^{\prime}} \Gamma^{2^{\prime}} \Gamma^{3^{\prime}} \not k\right)_{A B} .
\end{aligned}
$$

For the massive case we have the following modification of the polarization tensors

$$
\begin{aligned}
\varepsilon_{M N, \tilde{M} \tilde{N}}^{k_{i}} & =(-1)^{\sigma_{k_{i}}(\tilde{M})+\sigma_{k_{i}}(\tilde{N})} \varepsilon_{\mathrm{T}, M N, \tilde{M} \tilde{N}}^{k_{i}}, \\
\alpha_{M N P, \tilde{M} \tilde{N} \tilde{P}}^{k_{i}} & =(-1)^{\sigma_{k_{i}}(\tilde{M})+\sigma_{k_{i}}(\tilde{N})+\sigma_{k_{i}}(\tilde{P})} \alpha_{\mathrm{T}, M N P, \tilde{M} \tilde{N} \tilde{P}}^{k_{i}}, \\
t_{\tilde{M} A, M B}^{k_{i}} & =(-1)^{\sigma_{k_{i}}(\tilde{M})}\left(\frac{\alpha^{\prime}}{2}\right)^{1 / 2}\left(\mathcal{C}^{\dagger} i \Gamma^{0^{\prime}} \Gamma^{1^{\prime}} \Gamma^{2^{\prime}} \Gamma^{3^{\prime}} \not k\left(\hat{\eta}^{M \tilde{M}}-\frac{1}{9} \hat{\Gamma}^{\tilde{M}} \hat{\Gamma}^{M}\right)\right)_{A B} .
\end{aligned}
$$

Here we defined the twist factor

$$
\sigma_{k_{i}}(M)= \begin{cases}1 & \text { if } M=0^{\prime}, \ldots, 3^{\prime} \\ 0 & \text { if } M=4^{\prime}, 5, \ldots, 9\end{cases}
$$

and $0^{\prime}, \ldots, 3^{\prime}$ represent the AdS directions perpendicular to $k_{i}$. It will be useful computationally to consider the relation

$$
(-1)^{\sigma_{k_{i}}(M)} \eta^{M N}=(-1)^{\sigma(M)} \eta^{M N}-2 \frac{k_{i, A}^{M} k_{i, A}^{N}}{\Delta_{i}^{2}},
$$


where we introduce

$$
\sigma(M)= \begin{cases}1 & \text { if } M=0, \ldots, 4, \\ 0 & \text { if } M=5, \ldots, 9,\end{cases}
$$

and where we denote by $k_{i, \mathrm{~A}}=\left(k_{i}^{0}, \ldots, k_{i}^{4}, 0, \ldots, 0\right)$ the projection of $k_{i}$ onto the $\mathrm{AdS}_{5}$ part, and $-\Delta_{i}^{2}=k_{i, \mathrm{~A}}^{2}$. We then have the following useful relations

$$
\begin{aligned}
i \Gamma^{0^{\prime}} \Gamma^{1^{\prime}} \Gamma^{2^{\prime}} \Gamma^{3^{\prime}} & =\frac{1}{\Delta_{i}} \Gamma^{0} \Gamma^{1} \Gamma^{2} \Gamma^{3} \Gamma^{4} \not k_{i, A}, \\
\Gamma^{4} \Gamma^{3} \Gamma^{2} \Gamma^{1} \Gamma^{0}\left(\Gamma^{M_{1}} \ldots \Gamma^{M_{n}}\right) \Gamma^{0} \Gamma^{1} \Gamma^{2} \Gamma^{3} \Gamma^{4} & =(-1)^{1+n+\sigma\left(M_{1}\right)+\cdots+\sigma\left(M_{n}\right)} \Gamma^{M_{1}} \ldots \Gamma^{M_{n}} .
\end{aligned}
$$

Note that for the untwisted case, the same sixteen charges $Q_{\mathrm{L}, \alpha \tilde{a}}-i Q_{\mathrm{R}, \alpha \tilde{a}}$ and $Q_{\mathrm{L}, \dot{\alpha}}^{\tilde{a}}+i Q_{\mathrm{R}, \dot{\alpha}}^{\tilde{a}}$ annihilate both the vertex operator and its adjoint.

\section{Vertex function contractions}

\section{C.1 Fermion VEVs}

When computing correlators involving $\mathrm{R}-\mathrm{R}$ vertices, we will need vacuum expectation values for a number of fermion field combinations. Here we precompute these vacuum expectation values, using bosonization as outlined in section $\mathrm{A} .{ }^{13}$ In the following, it is assumed that factors $\psi^{M}(\psi \Theta)^{A}$ get contracted with massive polarization tensors $t_{k, \tilde{M} \tilde{A}, M A}(2.36)$, which obey

$$
t_{k, \tilde{M} \tilde{A}, M A}\left(\Gamma^{M}\right)_{B}^{A}=0, \quad\left(\Gamma^{\tilde{M}} C\right)^{\tilde{A} \tilde{B}} t_{k, \tilde{M} \tilde{B}, M B}=0 .
$$

These relations hold for the twisted as well as for the untwisted tensors. In computing the below correlators, we have added terms that vanish by these relations in order to obtain a uniform dependence on the worldsheet coordinates. It is also assumed that the term $\psi^{M} \psi^{N} \psi^{P}$ is always contracted with the polarization tensor (2.32), such that all terms symmetric in $M, N, P$ are discarded. In the relations below, the " $\simeq$ " signs mean that the respective relations only hold under these assumptions. The relevant VEVs are ${ }^{14}$

$$
\begin{aligned}
& \left\langle\psi_{1}^{M} e^{-\phi_{1}} \Theta_{2}^{A} e^{-\phi_{2} / 2} \Theta_{3}^{B} e^{-\phi_{3} / 2}\right\rangle=\frac{1}{\sqrt{2} z_{12} z_{13} z_{23}}\left(\Gamma^{M} C\right)^{A B}, \\
& \left\langle\psi_{1}^{M} \psi_{1}^{N} \psi_{1}^{P} e^{-\phi_{1}} \Theta_{2}^{A} e^{-\phi_{2} / 2} \Theta_{3}^{B} e^{-\phi_{3} / 2}\right\rangle=\frac{-1}{2 \sqrt{2} z_{12}^{2} z_{13}^{2}}\left(\Gamma^{M N P} C\right)^{A B}, \\
& \left\langle\psi_{1}^{M} e^{-\phi_{1}} \psi_{2}^{N}\left(\psi_{2} \Theta_{2}\right)^{A} e^{-\phi_{2} / 2} \Theta_{3}^{B} e^{-\phi_{3} / 2}\right\rangle \simeq \frac{2 \sqrt{2}}{5 z_{12}^{2} z_{23}^{2}}\left(\Gamma^{M N} C+8 \eta^{M N} C\right)^{A B}, \\
& \left\langle\psi_{1}^{M} \psi_{1}^{N} \psi_{1}^{P} e^{-\phi_{1}} \psi_{2}^{Q}\left(\psi_{2} \Theta_{2}\right)^{A} e^{-\phi_{2} / 2} \Theta_{3}^{B} e^{-\phi_{3} / 2}\right\rangle \simeq \frac{-\sqrt{2}}{5 z_{12}^{3} z_{13} z_{23}}\left(3 \Gamma^{M N P Q} C+12 \eta^{M Q} \Gamma^{N P} C\right)^{A B},
\end{aligned}
$$

\footnotetext{
${ }^{13}$ We thank Oliver Schlotterer for mentioning that VEVs involving terms $\psi^{M}(\psi \Theta)^{A}$ can alternatively be computed using the "excited spin fields" described in [52].

${ }^{14}$ The subscripts $1,2,3$ on the fields indicate dependence on the worldsheet coordinate $z_{1,2,3}$. We assume that all spin fields $\Theta^{A}$ have positive chirality, and we use the shorthand notation $\Gamma^{M N P \cdots}$ for $\Gamma^{M} \Gamma^{N} \Gamma^{P} \ldots$
} 


$$
\begin{aligned}
& \left\langle\psi_{1}^{M} e^{-\phi_{1}} \psi_{2}^{N}\left(\psi_{2} \Theta_{2}\right)^{A} e^{-\phi_{2} / 2} \psi_{3}^{P}\left(\psi_{3} \Theta_{3}\right)^{B} e^{-\phi_{3} / 2}\right\rangle \simeq \frac{16 \sqrt{2}}{25 z_{12} z_{13} z_{23}^{3}} \cdot \\
& \cdot\left(3 \Gamma^{M N P} C-\eta^{M N} \Gamma^{P} C+5 \eta^{M P} \Gamma^{N} C-25 \eta^{N P} \Gamma^{M} C\right)^{A B} \\
& \left\langle\psi_{1}^{M} \psi_{1}^{N} \psi_{1}^{P} e^{-\phi_{1}} \psi_{2}^{Q}\left(\psi_{2} \Theta_{2}\right)^{A} e^{-\phi_{3} / 2} \psi_{3}^{R}\left(\psi_{3} \Theta_{3}\right)^{B} e^{-\phi_{3} / 2}\right\rangle \simeq \frac{-64 \sqrt{2}}{5 z_{12}^{2} z_{13}^{2} z_{23}^{2}} \cdot \\
& \cdot\left(\frac{1}{40} \Gamma^{M N P Q R} C-\frac{3}{8} \eta^{M R} \Gamma^{N P Q} C-\frac{21}{40} \eta^{M Q} \Gamma^{N P R} C-\frac{5}{8} \eta^{Q R} \Gamma^{M N P} C-6 \eta^{M Q} \eta^{N R} \Gamma^{P} C\right)^{A B} \cdot
\end{aligned}
$$

\section{C.2 Contractions with three chiral primaries}

In a three-point function of three massless vertices $W_{k_{1,2,3}}$, we have $k_{1}^{2}=k_{2}^{2}=k_{3}^{2}=0$ and $k_{i} \cdot k_{j}=0$ : all scalar products among the momenta vanish.

$\left\langle\boldsymbol{W}_{\mathbf{1}} \boldsymbol{W}_{\mathbf{1}} \boldsymbol{W}_{\mathbf{1}}\right\rangle$. The correlator of three scalar level-zero NS-NS vertices $W_{1}(2.21),(2.22)$ reads $^{15}$

$$
\begin{gathered}
\left\langle W_{1, k_{1}}^{(-1,-1)}\left(z_{1}, \bar{z}_{1}\right) W_{1, k_{2}}^{(-1,-1)}\left(z_{2}, \bar{z}_{2}\right) W_{1, k_{3}}^{(0,0)}\left(z_{3}, \bar{z}_{3}\right)\right\rangle=-g_{\mathrm{c}}^{3} \delta\left(\sum_{j} k_{j}\right) \cdot \\
\cdot \varepsilon_{k_{1}, M \tilde{M}} \varepsilon_{k_{2}, N \tilde{N}} \varepsilon_{k_{3}, P \tilde{P}} \frac{\alpha^{\prime}}{2}\left(\eta^{M N} k_{1}^{P}-k_{1}^{N} \eta^{M P}+k_{2}^{M} \eta^{N P}\right)\left(\eta^{\tilde{M} \tilde{N}} k_{1}^{\tilde{P}}-k_{1}^{\tilde{N}} \eta^{\tilde{M} \tilde{P}}+k_{2}^{\tilde{M}} \eta^{\tilde{N} \tilde{P}}\right) .
\end{gathered}
$$

Using the twisted polarization tensors $\varepsilon_{\mathrm{T}}$, the result vanishes because all scalar products $k_{i} \cdot k_{j}$ among the three momenta vanish. But with untwisted polarization tensors, the result becomes

$$
\begin{aligned}
& \left\langle W_{1, k_{1}}\left(z_{1}, \bar{z}_{1}\right) W_{1, k_{2}}\left(z_{2}, \bar{z}_{2}\right) W_{1, k_{3}}\left(z_{3}, \bar{z}_{3}\right)\right\rangle \\
& \quad=g_{\mathrm{c}}^{3} \delta\left(\sum_{j} k_{j}\right) \frac{\alpha^{\prime}}{2} \frac{-8\left(J_{1}^{4}+6 J_{1}^{2} J_{2}^{2}+J_{2}^{4}+6 J_{1}^{2} J_{3}^{2}+6 J_{2}^{2} J_{3}^{2}+J_{3}^{4}\right) \tilde{\alpha}_{1} \tilde{\alpha}_{2} \tilde{\alpha}_{3} \tilde{\Sigma}}{J_{1}^{2} J_{2}^{2} J_{3}^{2}}
\end{aligned}
$$

where $\tilde{\alpha}_{j}, \tilde{\Sigma}$ are defined in $(2.2)$, and where $J_{j}=\sqrt{\left(k_{j}-k_{j, \mathrm{~A}}\right)^{2}}$ is the R-charge of the operator $W_{1, k_{j}} \cdot{ }^{16}$

$\left\langle\boldsymbol{W}_{\mathbf{1}} \boldsymbol{W}_{\mathbf{2}} \boldsymbol{W}_{\mathbf{2}}\right\rangle$. Using the VEV (C.2), the correlator of one flat-space scalar level-zero NS-NS vertex $W_{1}(2.21)$ with two level-zero R-R vertices $W_{2}(2.23)$ reads

$$
\begin{aligned}
\left\langle W_{1, k_{1}}^{(-1,-1)}\left(z_{1}, \bar{z}_{1}\right)\right. & \left.W_{2, k_{2}}^{(-1 / 2,-1 / 2)}\left(z_{2}, \bar{z}_{2}\right) W_{2, k_{3}}^{(-1 / 2,-1 / 2)}\left(z_{3}, \bar{z}_{3}\right)\right\rangle \\
= & -g_{\mathrm{c}}^{3} \delta\left(\sum_{j} k_{j}\right) \varepsilon_{k_{1}, M \tilde{M}} t_{k_{2}, A B} t_{k_{3}, C D} \frac{1}{2}\left(\Gamma^{M} C\right)^{B D}\left(\Gamma^{N} C\right)^{A C} .
\end{aligned}
$$

\footnotetext{
${ }^{15}$ Here and everywhere below, we do not write the fermionic ghosts $c, \tilde{c}$ explicitly, but we include their effect in the correlators.

${ }^{16}$ As before, we denote by $k_{j, \mathrm{~A}}=\left(k_{j}^{0}, \ldots, k_{j}^{4}, 0, \ldots, 0\right)$ the projection of $k_{j}$ onto the $\mathrm{AdS}_{5}$ part.
} 
Using the twisted polarization tensors $\varepsilon_{\mathrm{T}}, t_{\mathrm{T}}$, the result is zero again, while with untwisted polarizations it becomes ${ }^{17}$

$$
\begin{aligned}
& \left\langle W_{1, k_{1}}\left(z_{1}, \bar{z}_{1}\right) W_{2, k_{2}}\left(z_{2}, \bar{z}_{2}\right) W_{2, k_{3}}\left(z_{3}, \bar{z}_{3}\right)\right\rangle \\
& =-g_{\mathrm{c}}^{3} \delta\left(\sum_{j} k_{j}\right) \varepsilon_{\mathrm{T}, k_{1}, M \tilde{M}} \frac{\alpha^{\prime}}{2} \frac{1}{\Delta_{2} \Delta_{3}} \frac{1}{2} \operatorname{Tr}\left(\Gamma^{M} \not k_{3} \not k_{3, \mathrm{~A}} \Gamma^{43210} \Gamma^{\tilde{M}} \Gamma^{01234} \not k_{2, \mathrm{~A}} k_{2}\right) \\
& =-g_{\mathrm{c}}^{3} \delta\left(\sum_{j} k_{j}\right) \varepsilon_{\mathrm{T}, k_{1}, M \tilde{M}} \frac{\alpha^{\prime}}{2} \frac{1}{\Delta_{2} \Delta_{3}} \frac{1}{2}(-1)^{\sigma(\tilde{M})} \operatorname{Tr}\left(\Gamma^{M} \not k_{3} \not k_{3, \mathrm{~A}} \Gamma^{\tilde{M}} \not k_{2, \mathrm{~A}} \not k_{2}\right) \\
& =g_{\mathrm{c}}^{3} \delta\left(\sum_{j} k_{j}\right) \frac{\alpha^{\prime}}{2} \frac{-64\left(3 J_{1}^{2}+J_{2}^{2}+J_{3}^{2}\right) \tilde{\alpha}_{1} \tilde{\alpha}_{2} \tilde{\alpha}_{3} \tilde{\Sigma}}{J_{1}^{2} J_{2} J_{3}} .
\end{aligned}
$$

$\langle\boldsymbol{W} \boldsymbol{W} \boldsymbol{W}\rangle$. We are now in a position to assemble the correlator of three massless chiral primaries. When all three operators are twisted the result vanishes trivially since all scalar products among the momenta vanish. As a check, we can also compute the correlator with two twisted operators and again one obtains that it vanishes, which is due to a supersymmetric Ward identity. Finally the correlator for three primaries (3.5) yields (3.8)

$$
\left\langle W_{k_{1}}\left(z_{1}, \bar{z}_{1}\right) W_{k_{2}}\left(z_{2}, \bar{z}_{2}\right) W_{k_{3}}\left(z_{3}, \bar{z}_{3}\right)\right\rangle=g_{\mathrm{c}}^{3} \delta\left(\sum_{j} k_{j}\right) \frac{\alpha^{\prime}}{2} \frac{2 \tilde{\alpha}_{1} \tilde{\alpha}_{2} \tilde{\alpha}_{3} \tilde{\Sigma}^{5}}{J_{1}^{2} J_{2}^{2} J_{3}^{2}} .
$$

\section{C.3 Contractions with two chiral primaries}

With two massless vertices, $W_{k_{1,2}}$ and the level-one vertex $V_{k_{3}}$ we have $k_{1}^{2}=k_{2}^{2}=0$, $k_{3}^{2}=-4 / \alpha^{\prime}, k_{1,2} \cdot k_{3}=2 / \alpha^{\prime}$ and $k_{1} \cdot k_{2}=-2 / \alpha^{\prime}$.

$\left\langle\boldsymbol{W}_{\mathbf{1}} \boldsymbol{W}_{\mathbf{1}} \boldsymbol{V}_{\mathbf{1}}\right\rangle$. Both the left and right moving part of the correlator $\left\langle W_{1, k_{1}}^{(-1,-1)} W_{1, k_{2}}^{(-1,-1)} V_{1, k_{3}}^{(0,0)}\right\rangle$ are split into three parts corresponding to the terms in $V_{1, k_{3}}^{(0,0)}$. Using the symmetries of the polarization tensors, combining the three parts and multiplying contractions of the left-moving with the right-moving part, we obtain ${ }^{18}$

$$
\begin{aligned}
\left\langle W_{1, k_{1}}^{(-1,-1)}\left(z_{1}, \bar{z}_{1}\right) W_{1, k_{2}}^{(-1,-1)}\right. & \left.\left(z_{2}, \bar{z}_{2}\right) V_{1, k_{3}}^{(0,0)}\left(z_{3}, \bar{z}_{3}\right)\right\rangle \\
& =-g_{\mathrm{c}}^{3} \delta\left(\sum_{j} k_{j}\right) \varepsilon_{M, \tilde{M}}^{k_{1}} \varepsilon_{N, \tilde{N}}^{k_{2}} \varepsilon_{R S, \tilde{R} \tilde{S}}^{k_{3}} X^{M N R S} X^{\tilde{M} \tilde{N} \tilde{R} \tilde{S}},
\end{aligned}
$$

where

$$
X^{M N R S}=\frac{\alpha^{\prime}}{2}\left(\eta^{M N} k_{1}^{R} k_{1}^{S}+\frac{2}{\alpha^{\prime}} \eta^{M S} \eta^{N R}+\left(\eta^{M R} k_{3}^{N}-\eta^{N R} k_{3}^{M}\right) k_{1}^{S}\right) .
$$

Expanding the twisted polarization tensors and performing the index contractions gives

$$
\left\langle W_{1, \mathrm{~T}, k_{1}}^{(-1,-1)}\left(z_{1}, \bar{z}_{1}\right) W_{1, \mathrm{~T}, k_{2}}^{(-1,-1)}\left(z_{2}, \bar{z}_{2}\right) V_{1, \mathrm{~T}, k_{3}}^{(0,0)}\left(z_{3}, \bar{z}_{3}\right)\right\rangle=-2^{2} \times 3^{2} g_{\mathrm{c}}^{3} \delta\left(\sum_{j} k_{j}\right) .
$$

When contracting with the untwisted polarization tensors, the result becomes a lengthy rational function of $\Delta_{1,2,3}$ that we do not quote here.

\footnotetext{
${ }^{17}$ Because the theory is chiral, we use $\operatorname{Tr}(\mathbf{1})=16$.

${ }^{18}$ We do not denote the fermionic ghosts $c, \tilde{c}$ explicitly, but include their effect in the result.
} 
$\left\langle\boldsymbol{W}_{\mathbf{1}} \boldsymbol{W}_{\mathbf{1}} \boldsymbol{V}_{\mathbf{2}}\right\rangle$. For the correlator $\left\langle W_{1, k_{1}}^{(-1,-1)} W_{1, k_{2}}^{(-1,-1)} V_{2, k_{3}}^{(0,0)}\right\rangle$, we have to consider only the first term of $V_{2, k_{3}}^{(0,0)}$ since for the second there are $\psi$ 's which cannot be contracted. Assuming antisymmetry of the polarization tensor $\alpha$, the result is

$$
\begin{aligned}
& \left\langle W_{1, k_{1}}^{(-1,-1)}\left(z_{1}, \bar{z}_{1}\right) W_{1, k_{2}}^{(-1,-1)}\left(z_{2}, \bar{z}_{2}\right) V_{2, k_{3}}^{(0,0)}\left(z_{3}, \bar{z}_{3}\right)\right\rangle \\
& =-g_{\mathrm{c}}^{3} \delta\left(\sum_{j} k_{j}\right) \varepsilon_{M, \tilde{M}}^{k_{1}} \varepsilon_{N, \tilde{N}}^{k_{2}} \alpha_{R S T, \tilde{R} \tilde{S} \tilde{T}}^{k_{3}} \frac{\alpha^{\prime}}{2} 36 \eta^{M T} \eta^{N S} k_{1}^{R} \eta^{\tilde{M} \tilde{T}} \eta^{\tilde{N} \tilde{S}} k_{1}^{\tilde{R}} .
\end{aligned}
$$

Expanding the twisted polarization tensors and performing the index contractions gives

$$
\left\langle W_{1, \mathrm{~T}, k_{1}}^{(-1,-1)}\left(z_{1}, \bar{z}_{1}\right) W_{1, \mathrm{~T}, k_{2}}^{(-1,-1)}\left(z_{2}, \bar{z}_{2}\right) V_{2, \mathrm{~T}, k_{3}}^{(0,0)}\left(z_{3}, \bar{z}_{3}\right)\right\rangle=-2^{2} \times 7 g_{\mathrm{c}}^{3} \delta\left(\sum_{j} k_{j}\right) .
$$

When contracting with the untwisted polarization tensors, the result again becomes a complicated rational function of $\Delta_{1,2,3}$ that we do not quote here.

$\left\langle\boldsymbol{V}_{\mathbf{1}} \boldsymbol{W}_{\mathbf{2}} \boldsymbol{W}_{\mathbf{2}}\right\rangle$. Using the VEV (C.2), we can compute the correlator

$$
\begin{aligned}
\left\langle V_{1, k_{1}}^{(-1,-1)}\left(z_{1}, \bar{z}_{1}\right)\right. & \left.W_{2, k_{2}}^{(-1 / 2,-1 / 2)}\left(z_{2}, \bar{z}_{2}\right) W_{2, k_{3}}^{(-1 / 2,-1 / 2)}\left(z_{3}, \bar{z}_{3}\right)\right\rangle \\
& =-g_{\mathrm{c}}^{3} \delta\left(\sum_{j} k_{j}\right) \varepsilon_{M N, \tilde{M} \tilde{N}}^{k_{1}} \frac{1}{2} \operatorname{Tr}\left(t^{k_{2}} X^{M N}\left(t^{k_{3}}\right)^{\top}\left(X^{\tilde{M} \tilde{N}}\right)^{\top}\right),
\end{aligned}
$$

where superscript ${ }^{\top}$ denotes transposition in spinor indices, and

$$
X^{M N}=\sqrt{\frac{\alpha^{\prime}}{2}} k_{2}^{N} \Gamma^{M} C
$$

are the left/right-moving field contractions. Expanding the twisted polarization tensors and performing the index contractions and matrix algebra gives

$$
\left\langle V_{1, \mathrm{~T}, k_{1}}^{(-1,-1)}\left(z_{1}, \bar{z}_{1}\right) W_{2, \mathrm{~T}, k_{2}}^{(-1 / 2,-1 / 2)}\left(z_{2}, \bar{z}_{2}\right) W_{2, \mathrm{~T}, k_{3}}^{(-1 / 2,-1 / 2)}\left(z_{3}, \bar{z}_{3}\right)\right\rangle=-2^{4} g_{\mathrm{c}}^{3} \delta\left(\sum_{j} k_{j}\right) .
$$

Contracting with the untwisted polarization tensors gives an expression that we will refrain from quoting here.

$\left\langle\boldsymbol{V}_{\mathbf{2}} \boldsymbol{W}_{\mathbf{2}} \boldsymbol{W}_{\mathbf{2}}\right\rangle$. Using the $\operatorname{VEV}$ (C.3), the correlator is given by

$$
\begin{aligned}
& \left\langle V_{2, k_{1}}^{(-1,-1)}\left(z_{1}, \bar{z}_{1}\right) W_{2, k_{2}}^{(-1 / 2,-1 / 2)}\left(z_{2}, \bar{z}_{2}\right) W_{2, k_{3}}^{(-1 / 2,-1 / 2)}\left(z_{3}, \bar{z}_{3}\right)\right\rangle \\
& =-g_{\mathrm{c}}^{3} \delta\left(\sum_{j} k_{j}\right) \alpha_{M N P, \tilde{M} \tilde{N} \tilde{P}}^{k_{1}} \frac{1}{2} \operatorname{Tr}\left(t^{k_{2}} X^{M N P}\left(t^{k_{3}}\right)^{\top}\left(X^{\tilde{M} \tilde{N} \tilde{P}}\right)^{\top}\right),
\end{aligned}
$$

where

$$
X^{M N P}=\frac{1}{2} \Gamma^{M} \Gamma^{N} \Gamma^{P} C
$$

are the left/right-moving field contractions. Expanding the twisted polarization tensors and performing the index contractions and matrix algebra gives

$$
\left\langle V_{2, \mathrm{~T}, k_{1}}^{(-1,-1)}\left(z_{1}, \bar{z}_{1}\right) W_{2, \mathrm{~T}, k_{2}}^{(-1 / 2,-1 / 2)}\left(z_{2}, \bar{z}_{2}\right) W_{2, \mathrm{~T}, k_{3}}^{(-1 / 2,-1 / 2)}\left(z_{3}, \bar{z}_{3}\right)\right\rangle=-2^{4} \times 7 g_{\mathrm{c}}^{3} \delta\left(\sum_{j} k_{j}\right) .
$$

Once again, contracting with the untwisted polarization tensors gives a complicated expression that we will not write here. 
$\left\langle\boldsymbol{W}_{\mathbf{1}} \boldsymbol{W}_{\mathbf{2}} \boldsymbol{V}_{\mathbf{3}}\right\rangle$. Using the VEVs (C.2), (C.4), we compute the last correlator

$$
\begin{aligned}
\left\langle W_{1, k_{1}}^{(-1,-1)}\left(z_{1}, \bar{z}_{1}\right) W_{2, k_{2}}^{(-1 / 2,-1 / 2)}\left(z_{2}, \bar{z}_{2}\right) V_{3, k_{3}}^{(-1 / 2,-1 / 2)}\left(z_{3}, \bar{z}_{3}\right)\right\rangle & \\
& =-g_{\mathrm{c}}^{3} \delta\left(\sum_{j} k_{j}\right) \varepsilon_{M, \tilde{M}}^{k_{1}} \frac{1}{2} \operatorname{Tr}\left(t^{k_{2}} X^{M N}\left(t_{\tilde{N}, N}^{k_{3}}\right)^{\top}\left(X^{\tilde{M} \tilde{N}}\right)^{\top}\right),
\end{aligned}
$$

where

$$
X^{M N}=\sqrt{\frac{\alpha^{\prime}}{2}}\left(k_{1}^{N} \Gamma^{M} C+\eta^{M N} \not k_{3} C\right)
$$

are the left/right-moving field contractions, which have been simplified using (C.1) as well as the fact that all contractions of $k_{j}$ with $\varepsilon_{k_{j}}, t_{k_{j}}$ vanish. Expanding the twisted polarization tensors and performing the index contractions and matrix algebra gives

$$
\left\langle W_{1, \mathrm{~T}, k_{1}}^{(-1,-1)}\left(z_{1}, \bar{z}_{1}\right) W_{2, \mathrm{~T}, k_{2}}^{(-1 / 2,-1 / 2)}\left(z_{2}, \bar{z}_{2}\right) V_{3, \mathrm{~T}, k_{3}}^{(-1 / 2,-1 / 2)}\left(z_{3}, \bar{z}_{3}\right)\right\rangle=2^{7} g_{\mathrm{c}}^{3} \delta\left(\sum_{j} k_{j}\right) .
$$

Contracting with the untwisted polarization tensors gives a lengthy expression that we will refrain from writing here.

$\langle\boldsymbol{W} \boldsymbol{W} \boldsymbol{V}\rangle$. We can now assemble the different parts of the correlator with two massless vertices $W(3.5)$ and one massive vertex $V$ (3.10). As a check, we first compute the correlators with two and three twisted vertex operators, and we obtain that all of them vanish, which is explained by a supersymmetric Ward identity. For three untwisted vertices, meaning they are all primaries, all the complicated results of the different parts combine into the simple expression (3.11)

$$
\left\langle W_{k_{1}}\left(z_{1}, \bar{z}_{1}\right) W_{k_{2}}\left(z_{2}, \bar{z}_{2}\right) V_{k_{3}}\left(z_{3}, \bar{z}_{3}\right)\right\rangle=g_{\mathrm{c}}^{3} \delta\left(\sum_{j} k_{j}\right) \alpha^{\prime 2} \frac{\alpha_{1}^{2} \alpha_{2}^{2} \Sigma^{4} \tilde{\alpha}_{3}^{2} \tilde{\Sigma}^{2}}{\Delta_{1}^{2} \Delta_{2}^{2} \Delta_{3}^{4}}
$$

\section{C.4 Contractions with one chiral primary}

With two level-one vertices, $V_{k_{1,2}}$, and the massless vertex $W_{k_{3}}$ we have $k_{1}^{2}=k_{2}^{2}=-4 / \alpha^{\prime}$, $k_{3}^{2}=0, k_{1,2} \cdot k_{3}=0$ and $k_{1} \cdot k_{2}=4 / \alpha^{\prime}$.

$\left\langle\boldsymbol{V}_{\mathbf{1}} \boldsymbol{V}_{\mathbf{1}} \boldsymbol{W}_{\mathbf{1}}\right\rangle$. The left and right-moving parts of the correlator $\left\langle V_{1, k_{1}}^{(-1,-1)} V_{1, k_{2}}^{(-1,-1)} W_{1, k_{3}}^{(0,0)}\right\rangle$ are split into two parts corresponding to the terms in $W_{1, k_{3}}^{(0,0)}$. Using the symmetries of the polarization tensors, combining those parts and multiplying left and right-moving contractions we obtain ${ }^{19}$

$$
\begin{aligned}
& \left\langle V_{1, k_{1}}^{(-1,-1)}\left(z_{1}, \bar{z}_{1}\right) V_{1, k_{2}}^{(-1,-1)}\left(z_{2}, \bar{z}_{2}\right) W_{1, k_{3}}^{(0,0)}\left(z_{3}, \bar{z}_{3}\right)\right\rangle \\
& =-g_{\mathrm{c}}^{3} \delta\left(\sum_{j} k_{j}\right) \varepsilon_{M N, \tilde{M} \tilde{N}}^{k_{1}} \varepsilon_{P Q, \tilde{P} \tilde{Q}}^{k_{2}} \varepsilon_{R, \tilde{R}}^{k_{3}} X^{M N P Q R} X^{\tilde{M} \tilde{N} \tilde{P} \tilde{Q} \tilde{R}}
\end{aligned}
$$

where

$$
\begin{aligned}
X^{M N P Q R}= & \sqrt{\frac{\alpha^{\prime}}{2}} \eta^{M P}\left(\eta^{N Q} k_{1}^{R}+2 \eta^{Q R} k_{2}^{N}+2 \eta^{N R} k_{3}^{Q}+\frac{\alpha^{\prime}}{2} k_{2}^{N} k_{3}^{Q} k_{1}^{R}\right) \\
& +\left(\frac{\alpha^{\prime}}{2}\right)^{3 / 2}\left(\eta^{M R} k_{3}^{P}+\eta^{P R} k_{2}^{M}\right) k_{2}^{N} k_{3}^{Q} .
\end{aligned}
$$

\footnotetext{
${ }^{19}$ Once again, we do not denote the fermionic ghosts $c, \tilde{c}$ explicitly, but include their effect in the result.
} 
Expanding the twisted polarization tensors and performing the index contractions gives

$$
\left\langle V_{1, \mathrm{~T}, k_{1}}^{(-1,-1)}\left(z_{1}, \bar{z}_{1}\right) V_{1, \mathrm{~T}, k_{2}}^{(-1,-1)}\left(z_{2}, \bar{z}_{2}\right) W_{1, \mathrm{~T}, k_{3}}^{(0,0)}\left(z_{3}, \bar{z}_{3}\right)\right\rangle=2^{3} \times 11 g_{\mathrm{c}}^{3} \delta\left(\sum_{j} k_{j}\right) .
$$

When contracting with the untwisted polarization tensors, the result becomes a lengthy rational function of $\Delta_{1,2,3}$ that we do not quote here.

$\left\langle\boldsymbol{V}_{\mathbf{2}} \boldsymbol{V}_{\mathbf{2}} \boldsymbol{W}_{\mathbf{1}}\right\rangle$. As before, there are two parts in the correlator $\left\langle V_{2, k_{1}}^{(-1,-1)} V_{2, k_{2}}^{(-1,-1)} W_{1, k_{3}}^{(0,0)}\right\rangle$. Assuming antisymmetry of the polarization tensor $\alpha$, they combine into

$$
\begin{gathered}
\left\langle V_{2, k_{1}}^{(-1,-1)}\left(z_{1}, \bar{z}_{1}\right) V_{2, k_{2}}^{(-1,-1)}\left(z_{2}, \bar{z}_{2}\right) W_{1, k_{3}}^{(0,0)}\left(z_{3}, \bar{z}_{3}\right)\right\rangle=-g_{\mathrm{c}}^{3} \delta\left(\sum_{j} k_{j}\right) \alpha_{M N P, \tilde{M} \tilde{N} \tilde{N}}^{k_{1}} \alpha_{Q R S, \tilde{Q} \tilde{R} \tilde{S}}^{k_{2}} \varepsilon^{k_{3}} \cdot \\
\cdot \frac{\alpha^{\prime}}{2} 36 \eta^{N S} \eta^{P R}\left(k_{1}^{T} \eta^{M Q}+3\left(\eta^{M T} k_{3}^{Q}-\eta^{Q T} k_{3}^{M}\right)\right) \eta^{\tilde{N} \tilde{S}} \eta^{\tilde{P} \tilde{R}}\left(k_{1}^{\tilde{T}} \eta^{\tilde{M} \tilde{Q}}+3\left(\eta^{\tilde{M} \tilde{T}} k_{3}^{\tilde{Q}}-\eta^{\tilde{Q} \tilde{T}} k_{3}^{\tilde{M}}\right)\right) .
\end{gathered}
$$

Expanding the twisted polarization tensors and performing the index contractions gives

$$
\left\langle V_{2, \mathrm{~T}, k_{1}}^{(-1,-1)}\left(z_{1}, \bar{z}_{1}\right) V_{2, \mathrm{~T}, k_{2}}^{(-1,-1)}\left(z_{2}, \bar{z}_{2}\right) W_{1, \mathrm{~T}, k_{3}}^{(0,0)}\left(z_{3}, \bar{z}_{3}\right)\right\rangle=2^{3} \times 3 \times 7 g_{\mathrm{c}}^{3} \delta\left(\sum_{j} k_{j}\right) .
$$

Contracting with the untwisted polarization tensors, the result becomes a complicated function of $\Delta_{1,2,3}$ that we will not quote here.

$\left\langle\boldsymbol{V}_{\mathbf{2}} \boldsymbol{V}_{\mathbf{1}} \boldsymbol{W}_{\mathbf{1}}\right\rangle$. In the case of $\left\langle V_{2, k_{1}}^{(-1,-1)} V_{1, k_{2}}^{(-1,-1)} W_{1, k_{3}}^{(0,0)}\right\rangle$, we have to consider only the second term of $W_{1, k_{3}}^{(0,0)}$ since for the first there are $\psi$ 's which cannot be contracted. Then, assuming antisymmetry of the polarization tensor $\alpha$, the correlator is given by

$$
\begin{aligned}
& \left\langle V_{2, k_{1}}^{(-1,-1)}\left(z_{1}, \bar{z}_{1}\right) V_{1, k_{2}}^{(-1,-1)}\left(z_{2}, \bar{z}_{2}\right) W_{1, k_{3}}^{(0,0)}\left(z_{3}, \bar{z}_{3}\right)\right\rangle \\
= & -g_{\mathrm{c}}^{3} \delta\left(\sum_{j} k_{j}\right) \alpha_{M N P, \tilde{M} \tilde{N} \tilde{N}}^{k_{1}} \varepsilon_{R S, \tilde{R} \tilde{S}}^{k_{2}} \varepsilon_{T, \tilde{T}}^{k_{3}}\left(\frac{\alpha^{\prime}}{2}\right)^{2} 36 k_{3}^{S} k_{3}^{N} \eta^{M T} \eta^{P R} k_{3}^{\tilde{S}} k_{3}^{\tilde{N}} \eta^{\tilde{M} \tilde{T}} \eta^{\tilde{P} \tilde{R}} .
\end{aligned}
$$

Expanding the twisted polarization tensors and performing the index contractions gives

$$
\left\langle V_{2, \mathrm{~T}, k_{1}}^{(-1,-1)}\left(z_{1}, \bar{z}_{1}\right) V_{1, \mathrm{~T}, k_{2}}^{(-1,-1)}\left(z_{2}, \bar{z}_{2}\right) W_{1, \mathrm{~T}, k_{3}}^{(0,0)}\left(z_{3}, \bar{z}_{3}\right)\right\rangle=0 .
$$

As before, we do not quote the result of contracting with the untwisted polarization tensors.

$\left\langle\boldsymbol{V}_{\mathbf{1}} \boldsymbol{W}_{\mathbf{2}} \boldsymbol{V}_{\mathbf{3}}\right\rangle$. Using the VEVs (C.2), (C.4), we can compute the correlator

$$
\begin{aligned}
& \left\langle V_{1, k_{1}}^{(-1,-1)}\left(z_{1}, \bar{z}_{1}\right) W_{2, k_{2}}^{(-1 / 2,-1 / 2)}\left(z_{2}, \bar{z}_{2}\right) V_{3, k_{3}}^{(-1 / 2,-1 / 2)}\left(z_{3}, \bar{z}_{3}\right)\right\rangle \\
& =-g_{\mathrm{c}}^{3} \delta\left(\sum_{j} k_{j}\right) \varepsilon_{M N, \tilde{M} \tilde{N}}^{k_{1}} \frac{1}{2} \operatorname{Tr}\left(t^{k_{2}} X^{M N P}\left(t_{\tilde{P}, P}^{k_{3}}\right)^{\top}\left(X^{\tilde{M} \tilde{N} \tilde{P}}\right)^{\top}\right),
\end{aligned}
$$

where

$$
X^{M N P}=\left(\eta^{N P}+\frac{\alpha^{\prime}}{2} k_{2}^{N} k_{1}^{P}\right) \Gamma^{M} C+\frac{\alpha^{\prime}}{2} k_{2}^{N} \eta^{M P} \not k_{3} C
$$

are the left/right-moving field contractions, which have been simplified using (C.1) as well as the symmetry of $\varepsilon_{k_{1}}$ and the fact that all contractions of $k_{j}$ with $\varepsilon_{k_{j}}, t_{k_{j}}$ vanish. 
Expanding the twisted polarization tensors and performing the index contractions and matrix algebra gives

$$
\left\langle V_{1, \mathrm{~T}, k_{1}}^{(-1,-1)}\left(z_{1}, \bar{z}_{1}\right) W_{2, \mathrm{~T}, k_{2}}^{(-1 / 2,-1 / 2)}\left(z_{2}, \bar{z}_{2}\right) V_{3, \mathrm{~T}, k_{3}}^{(-1 / 2,-1 / 2)}\left(z_{3}, \bar{z}_{3}\right)\right\rangle=0 .
$$

Once again, contracting with the untwisted polarization tensors gives an expression that we will refrain from quoting here.

$\left\langle\boldsymbol{V}_{\mathbf{2}} \boldsymbol{W}_{\mathbf{2}} \boldsymbol{V}_{\mathbf{3}}\right\rangle$. Using the VEVs (C.3), (C.5), the correlator is given by

$$
\begin{aligned}
& \left\langle V_{2, k_{1}}^{(-1,-1)}\left(z_{1}, \bar{z}_{1}\right) W_{2, k_{2}}^{(-1 / 2,-1 / 2)}\left(z_{2}, \bar{z}_{2}\right) V_{3, k_{3}}^{(-1 / 2,-1 / 2)}\left(z_{3}, \bar{z}_{3}\right)\right\rangle \\
& =-g_{\mathrm{c}}^{3} \delta\left(\sum_{j} k_{j}\right) \alpha_{M N P, \tilde{M} \tilde{N} \tilde{P}}^{k_{1}} \frac{1}{2} \operatorname{Tr}\left(t^{k_{2}} X^{M N P R}\left(t_{\tilde{R}, R}^{k_{3}}\right)^{\top}\left(X^{\tilde{M} \tilde{N} \tilde{P} \tilde{R}}\right)^{\top}\right),
\end{aligned}
$$

where

$$
X^{M N P R}=\sqrt{\frac{\alpha^{\prime}}{2}} \frac{1}{2} k_{1}^{R} \Gamma^{M} \Gamma^{N} \Gamma^{P} C+\sqrt{\frac{\alpha^{\prime}}{2}} \frac{3}{2} \eta^{M R} \Gamma^{N} \Gamma^{P} \not k_{3} C
$$

are the left/right-moving field contractions, which have been simplified using (C.1) as well as the symmetry of $\alpha_{k_{1}}$ and the fact that all contractions of $k_{j}$ with $\alpha_{k_{j}}, t_{k_{j}}$ vanish. Expanding the twisted polarization tensors and performing the index contractions and matrix algebra gives

$$
\left\langle V_{2, \mathrm{~T}, k_{1}}^{(-1,-1)}\left(z_{1}, \bar{z}_{1}\right) W_{2, \mathrm{~T}, k_{2}}^{(-1 / 2,-1 / 2)}\left(z_{2}, \bar{z}_{2}\right) V_{3, T, k_{3}}^{(-1 / 2,-1 / 2)}\left(z_{3}, \bar{z}_{3}\right)\right\rangle=0
$$

Contracting with the untwisted polarization tensors gives a complicated function of $\Delta_{1,2,3}$ that we will refrain from quoting here.

$\left\langle\boldsymbol{W}_{\mathbf{1}} \boldsymbol{V}_{\mathbf{3}} \boldsymbol{V}_{\mathbf{3}}\right\rangle$. Using the VEVs (C.2), (C.4), (C.6), the last correlator is

$$
\begin{aligned}
\left\langle W_{1, k_{1}}^{(-1,-1)}\left(z_{1}, \bar{z}_{1}\right)\right. & \left.V_{3, k_{2}}^{(-1 / 2,-1 / 2)}\left(z_{2}, \bar{z}_{2}\right) V_{3, k_{3}}^{(-1 / 2,-1 / 2)}\left(z_{3}, \bar{z}_{3}\right)\right\rangle \\
= & -g_{\mathrm{c}}^{3} \delta\left(\sum_{j} k_{j}\right) \varepsilon_{M, \tilde{M}}^{k_{1}} \frac{1}{2} \operatorname{Tr}\left(t_{\tilde{N}, N}^{k_{2}} X^{M N P}\left(t_{\tilde{P}, P}^{k_{3}}\right)^{\top}\left(X^{\tilde{M} \tilde{N} \tilde{P}}\right)^{\top}\right),
\end{aligned}
$$

where

$$
X^{M N P}=\left(\eta^{N P}+\frac{\alpha^{\prime}}{2} k_{3}^{N} k_{1}^{P}\right) \Gamma^{M} C+\frac{\alpha^{\prime}}{2} k_{3}^{N} \eta^{M P} \not k_{3} C-\frac{\alpha^{\prime}}{2} k_{1}^{P} \eta^{M N} \not k_{2} C+\frac{\alpha^{\prime}}{2} \frac{1}{2} \eta^{N P} \not k_{2} \Gamma^{M} \not k_{3} C
$$

are the left/right-moving field contractions, which have been simplified using (C.1) as well as the fact that all contractions of $k_{j}$ with $\varepsilon_{k_{j}}, t_{k_{j}}$ vanish. Expanding the twisted polarization tensors and performing the index contractions and matrix algebra gives

$$
\left\langle W_{1, \mathrm{~T}, k_{1}}^{(-1,-1)}\left(z_{1}, \bar{z}_{1}\right) V_{3, \mathrm{~T}, k_{2}}^{(-1 / 2,-1 / 2)}\left(z_{2}, \bar{z}_{2}\right) V_{3, \mathrm{~T}, k_{3}}^{(-1 / 2,-1 / 2)}\left(z_{3}, \bar{z}_{3}\right)\right\rangle=-2^{9} g_{\mathrm{c}}^{3} \delta\left(\sum_{j} k_{j}\right) .
$$

Again, we do not write here the result of contracting with the untwisted polarization tensors. 
$\langle\boldsymbol{V} \boldsymbol{V} \boldsymbol{W}\rangle$. We now can assemble all the different parts of the correlator of a massless vertex $W(3.5)$ and two massive vertices $V(3.10)$. When two or three of them are twisted, we find once again that the results vanish, which matches the supersymmetric Ward identity. For three primaries, the correlator becomes (3.14)

$$
\begin{aligned}
& \left\langle V_{k_{1}}\left(z_{1}, \bar{z}_{1}\right) V_{k_{2}}\left(z_{2}, \bar{z}_{2}\right) W_{k_{3}}\left(z_{3}, \bar{z}_{3}\right)\right\rangle=g_{\mathrm{c}}^{3} \delta\left(\sum_{j} k_{j}\right) \frac{\Sigma^{4}}{\Delta_{1}^{4} \Delta_{2}^{4} \Delta_{3}^{2}} . \\
& \cdot\left(-\frac{1}{2} \Omega_{1}^{2} \alpha_{3}^{4}+\alpha_{3}^{2}\left(3 \Omega_{1}^{2}+2 \Omega_{1} \alpha_{3}+\alpha_{3}^{2}\right) \Omega_{4}-\frac{1}{2} \Omega_{1}\left(\Omega_{1}-4 \alpha_{3}\right) \Omega_{4}^{2}+\Omega_{4}^{3}\right) .
\end{aligned}
$$

where

$$
\Omega_{1}=\alpha_{1}+\alpha_{2}, \quad \Omega_{4}=\alpha^{\prime} \alpha_{1} \alpha_{2} \alpha_{3} \Sigma .
$$

\section{C.5 Contractions with zero chiral primaries}

With three level-one vertices, we have $k_{j}^{2}=-4 / \alpha^{\prime}$, and $k_{i} \cdot k_{j}=2 / \alpha^{\prime}$ for $i \neq j$.

$\left\langle\boldsymbol{V}_{\mathbf{1}} \boldsymbol{V}_{\mathbf{1}} \boldsymbol{V}_{\mathbf{1}}\right\rangle$. The correlator of three vertices $V_{1}(2.29)$, (2.33) evaluates to ${ }^{20}$

$$
\begin{aligned}
\left\langle V_{1, k_{1}}^{(-1,-1)}\left(z_{1}, \bar{z}_{1}\right)\right. & \left.V_{1, k_{2}}^{(-1,-1)}\left(z_{2}, \bar{z}_{2}\right) V_{1, k_{3}}^{(0,0)}\left(z_{3}, \bar{z}_{3}\right)\right\rangle \\
= & -g_{\mathrm{c}}^{3} \delta\left(\sum_{j} k_{j}\right) \varepsilon_{M N, \tilde{M} \tilde{N}}^{k_{1}} \varepsilon_{P Q, \tilde{P} \tilde{Q}}^{k_{2}} \varepsilon_{R S, \tilde{R} \tilde{S}}^{k_{3}} X^{M N P Q R S} X^{\tilde{M} \tilde{N} \tilde{P} \tilde{Q} \tilde{R} \tilde{S}}
\end{aligned}
$$

where, using the antisymmetry of the polarization tensor $\varepsilon_{M N, \tilde{M} \tilde{N}}$ as well as $k_{j} \cdot \varepsilon^{k_{j}}=0$,

$$
\begin{aligned}
X^{M N P Q R S}=\frac{1}{2} \eta^{M P} & \left(\eta^{N R}\left(\eta^{Q S}-\frac{\alpha^{\prime}}{2} 3 k_{1}^{Q} k_{1}^{S}\right)-\frac{\alpha^{\prime}}{2}\left(\eta^{N Q}-\frac{\alpha^{\prime}}{2} k_{3}^{N} k_{3}^{Q}\right) k_{1}^{R} k_{2}^{S}\right) \\
& +\left(5 \text { permutations of }\left(\left(M, N, k_{1}\right),\left(P, Q, k_{2}\right),\left(R, S, k_{3}\right)\right)\right) .
\end{aligned}
$$

Contracting with the twisted polarization tensors $\varepsilon_{\mathrm{T}}(2.31)$ gives

$$
\left\langle V_{1, \mathrm{~T}, k_{1}}^{(-1,-1)}\left(z_{1}, \bar{z}_{1}\right) V_{1, \mathrm{~T}, k_{2}}^{(-1,-1)}\left(z_{2}, \bar{z}_{2}\right) V_{1, \mathrm{~T}, k_{3}}^{(0,0)}\left(z_{3}, \bar{z}_{3}\right)\right\rangle=-2^{4} \times 3 \times 53 g_{\mathrm{c}}^{3} \delta\left(\sum_{j} k_{j}\right) .
$$

When contracting with the untwisted polarization tensors, the result becomes a lengthy rational function of $\Delta_{1}, \Delta_{2}, \Delta_{3}$ (or equivalently $J_{1}, J_{2}, J_{3}$ ) that we do not quote here. For $J_{i} \ll \sqrt{1 / \alpha^{\prime}}$ (i.e. $\Delta_{i} \approx \sqrt{4 / \alpha^{\prime}}$ ), it becomes exactly (C.48), plus $\mathcal{O}\left(J_{i}^{2} \alpha^{\prime}\right)$ terms. This can be understood by noting that all terms in the contraction contain an even number of untwisted index pairs.

$\left\langle\boldsymbol{V}_{\mathbf{1}} \boldsymbol{V}_{\mathbf{1}} \boldsymbol{V}_{\mathbf{2}}\right\rangle$. In the correlator $\left\langle V_{1, k_{1}}^{(-1,-1)} V_{1, k_{2}}^{(-1,-1)} V_{2, k_{3}}^{(0,0)}\right\rangle$, only the first term in $V_{2, k_{3}}^{(0,0)}(2.34)$ contributes, since the second term yields an odd number of $\psi$ 's, which cannot be fully contracted. Assuming antisymmetry of the polarization tensor $\alpha$, the result is

$$
\begin{aligned}
& \left\langle V_{1, k_{1}}^{(-1,-1)}\left(z_{1}, \bar{z}_{1}\right) V_{1, k_{2}}^{(-1,-1)}\left(z_{2}, \bar{z}_{2}\right) V_{2, k_{3}}^{(0,0)}\left(z_{3}, \bar{z}_{3}\right)\right\rangle \\
& =-g_{\mathrm{c}}^{3} \delta\left(\sum_{j} k_{j}\right) \varepsilon_{M N, \tilde{M} \tilde{N}}^{k_{1}} \varepsilon_{P Q, \tilde{P} \tilde{Q}}^{k_{2}} \alpha_{R S T, \tilde{R} \tilde{S} \tilde{T}}^{k_{3}} X^{M N P Q R S T} X^{\tilde{M} \tilde{N} \tilde{P} \tilde{Q} \tilde{R} \tilde{S} \tilde{T}},
\end{aligned}
$$

\footnotetext{
${ }^{20}$ As before, we do not denote the fermionic ghosts $c, \tilde{c}$ explicitly, but include their effect in the result.
} 
where

$$
X^{M N P Q R S T}=\sqrt{\frac{\alpha^{\prime}}{2}} 6 \eta^{M R} \eta^{P S}\left(\eta^{N T} k_{1}^{Q}-\eta^{N Q} k_{1}^{T}-\eta^{Q T} k_{2}^{N}+\frac{\alpha^{\prime}}{2} k_{2}^{N} k_{1}^{Q} k_{1}^{T}\right) .
$$

Contracting with the twisted polarization tensors $\varepsilon_{\mathrm{T}}(2.31)$ and $\alpha_{\mathrm{T}}(2.32)$ gives

$$
\left\langle V_{1, \mathrm{~T}, k_{1}}^{(-1,-1)}\left(z_{1}, \bar{z}_{1}\right) V_{1, \mathrm{~T}, k_{2}}^{(-1,-1)}\left(z_{2}, \bar{z}_{2}\right) V_{2, \mathrm{~T}, k_{3}}^{(0,0)}\left(z_{3}, \bar{z}_{3}\right)\right\rangle=2 \times 3 \times 7^{2} g_{\mathrm{c}}^{3} \delta\left(\sum_{j} k_{j}\right) .
$$

The result for untwisted vertices with general $J_{i}$ is again lengthy and we refrain from quoting it. For $J_{i} \ll \sqrt{1 / \alpha^{\prime}}$, it becomes exactly the negative of (C.51), plus $\mathcal{O}\left(J_{i}^{2} \alpha^{\prime}\right)$ terms, which follows from the fact that all terms in the contraction contain an odd number of untwisted index pairs.

$\left\langle\boldsymbol{V}_{\mathbf{2}} \boldsymbol{V}_{\mathbf{2}} \boldsymbol{V}_{\mathbf{1}}\right\rangle$. Performing the field contractions, the correlator $\left\langle V_{2, k_{1}} V_{2, k_{2}} V_{1, k_{3}}\right\rangle$ becomes

$$
\begin{aligned}
& \left\langle V_{2, k_{1}}^{(-1,-1)}\left(z_{1}, \bar{z}_{1}\right) V_{2, k_{2}}^{(-1,-1)}\left(z_{2}, \bar{z}_{2}\right) V_{1, k_{3}}^{(0,0)}\left(z_{3}, \bar{z}_{3}\right)\right\rangle \\
& \quad=-g_{\mathrm{c}}^{3} \delta\left(\sum_{j} k_{j}\right) \alpha_{M N P, \tilde{M} \tilde{N} \tilde{P}}^{k_{1}} \alpha_{Q R S, \tilde{Q} \tilde{R} \tilde{S}}^{k_{2}} \varepsilon_{T U, \tilde{T} \tilde{U}}^{k_{3}} X^{M N P Q R S T U} X^{\tilde{M} \tilde{N} \tilde{P} \tilde{Q} \tilde{R} \tilde{S} \tilde{T} \tilde{U}},
\end{aligned}
$$

where

$$
X^{M N P Q R S T U}=6 \eta^{N R} \eta^{P S}\left(3 \eta^{M U}\left(\eta^{Q T}-\frac{\alpha^{\prime}}{2} k_{1}^{Q} k_{1}^{T}\right)+\frac{\alpha^{\prime}}{2}\left(\eta^{M Q} k_{1}^{U}+3 \eta^{Q U} k_{2}^{M}\right) k_{1}^{T}\right) .
$$

Expanding the twisted polarization tensors and performing the index contractions gives

$$
\left\langle V_{2, \mathrm{~T}, k_{1}}^{(-1,-1)}\left(z_{1}, \bar{z}_{1}\right) V_{2, \mathrm{~T}, k_{2}}^{(-1,-1)}\left(z_{2}, \bar{z}_{2}\right) V_{1, \mathrm{~T}, k_{3}}^{(0,0)}\left(z_{3}, \bar{z}_{3}\right)\right\rangle=-2^{2} \times 3 \times 7 \times 19 g_{\mathrm{c}}^{3} \delta\left(\sum_{j} k_{j}\right) .
$$

We refrain from writing out the lengthy result for untwisted vertices. Again, for $J_{i} \ll$ $\sqrt{1 / \alpha^{\prime}}$, it becomes exactly (C.54), plus $\mathcal{O}\left(J_{i}^{2} \alpha^{\prime}\right)$ terms, because each term contains an even number of untwisted index contractions.

$\left\langle\boldsymbol{V}_{\mathbf{2}} \boldsymbol{V}_{\mathbf{2}} \boldsymbol{V}_{\mathbf{2}}\right\rangle$. Performing the field contractions, the correlator $\left\langle V_{2, k_{1}} V_{2, k_{2}} V_{2, k_{3}}\right\rangle$ becomes (using the antisymmetry of the polarization tensors $\alpha$ )

$$
\begin{aligned}
& \left\langle V_{2, k_{1}}^{(-1,-1)}\left(z_{1}, \bar{z}_{1}\right) V_{2, k_{2}}^{(-1,-1)}\left(z_{2}, \bar{z}_{2}\right) V_{2, k_{3}}^{(0,0)}\left(z_{3}, \bar{z}_{3}\right)\right\rangle \\
= & -g_{\mathrm{c}}^{3} \delta\left(\sum_{j} k_{j}\right) \alpha_{M N P, \tilde{M} \tilde{N} \tilde{P}}^{k_{1}} \alpha_{Q R S, \tilde{Q} \tilde{R} \tilde{S}}^{k_{2}} \alpha_{T U V, \tilde{T} \tilde{U} \tilde{V}}^{k_{3}} X^{M N P Q R S T U V} X^{\tilde{M} \tilde{N} \tilde{P} \tilde{Q} \tilde{R} \tilde{S} \tilde{T} \tilde{V}},
\end{aligned}
$$

where

$$
X^{M N P Q R S T U V}=2^{2} 3^{3} \eta^{M Q} \eta^{S V} \sqrt{\frac{\alpha^{\prime}}{2}}\left(\eta^{N R} \eta^{P U} k_{1}^{T}+\eta^{N T}\left(\eta^{R U} k_{2}^{P}-\eta^{P U} k_{1}^{R}\right)\right) .
$$

Expanding the twisted polarization tensors and performing the index contractions gives

$$
\left\langle V_{2, \mathrm{~T}, k_{1}}^{(-1,-1)}\left(z_{1}, \bar{z}_{1}\right) V_{2, \mathrm{~T}, k_{2}}^{(-1,-1)}\left(z_{2}, \bar{z}_{2}\right) V_{2, \mathrm{~T}, k_{3}}^{(0,0)}\left(z_{3}, \bar{z}_{3}\right)\right\rangle=2 \times 3^{3} \times 7 \times 13 g_{\mathrm{c}}^{3} \delta\left(\sum_{j} k_{j}\right) .
$$

The result for untwisted vertices again reduces to the negative of (C.57) for $J_{i} \ll \sqrt{1 / \alpha^{\prime}}$, plus $\mathcal{O}\left(J_{i}^{2} \alpha^{\prime}\right)$ terms. 
$\left\langle\boldsymbol{V}_{\mathbf{1}} \boldsymbol{V}_{\mathbf{3}} \boldsymbol{V}_{\mathbf{3}}\right\rangle$. Using the VEVs (C.2), (C.4), (C.6), we can compute the correlator

$$
\begin{aligned}
& \left\langle V_{1, k_{1}}^{(-1,-1)}\left(z_{1}, \bar{z}_{1}\right) V_{3, k_{2}}^{(-1 / 2,-1 / 2)}\left(z_{2}, \bar{z}_{2}\right) V_{3, k_{3}}^{(-1 / 2,-1 / 2)}\left(z_{3}, \bar{z}_{3}\right)\right\rangle \\
& =-g_{\mathrm{c}}^{3} \delta\left(\sum_{j} k_{j}\right) \varepsilon_{M N, \tilde{M} \tilde{N}}^{k_{1}} t_{\tilde{P} \tilde{A}, P A}^{k_{2}} t_{\tilde{Q} \tilde{B}, Q B}^{k_{3}} X_{\mathrm{L}}^{M N P Q, A B} X_{\mathrm{R}}^{\tilde{M} \tilde{N} \tilde{P} \tilde{Q}, \tilde{A} \tilde{B}},
\end{aligned}
$$

where

$$
\begin{aligned}
& X_{\mathrm{L}, \mathrm{R}}^{M N P Q, A B}=\frac{\sqrt{\alpha^{\prime}}}{2}\left[\left(-\eta^{N P} k_{1}^{Q}+\eta^{N Q} k_{1}^{P}-\eta^{P Q} k_{2}^{N}+\frac{\alpha^{\prime}}{2} k_{1}^{Q} k_{1}^{P} k_{2}^{N}\right) \Gamma^{M} C\right. \\
& \left.\quad+\left(\eta^{N Q}+\frac{\alpha^{\prime}}{2} k_{1}^{Q} k_{2}^{N}\right) \eta^{M P} \not k_{2} C+\left(-\eta^{N P}+\frac{\alpha^{\prime}}{2} k_{1}^{P} k_{2}^{N}\right) \eta^{M Q} \not k_{3} C-\frac{\alpha^{\prime}}{2} \frac{1}{2} k_{2}^{N} \eta^{P Q} \not k_{2} \Gamma^{M} \not k_{3} C\right]^{A B}
\end{aligned}
$$

are the left/right-moving field contractions, which have been simplified using (C.1) as well as the symmetry of $\varepsilon_{k_{1}}$ and the fact that all contractions of $k_{j}$ with $\varepsilon_{k_{j}}, t_{k_{j}}$ vanish. Performing the matrix algebra and all the contractions, and using the twisted polarization tensors $t_{\mathrm{T}}, \varepsilon_{\mathrm{T}}$, the correlator evaluates to

$$
\left\langle V_{1, \mathrm{~T}, k_{1}}^{(-1,-1)}\left(z_{1}, \bar{z}_{1}\right) V_{3, \mathrm{~T}, k_{2}}^{(-1 / 2,-1 / 2)}\left(z_{2}, \bar{z}_{2}\right) V_{3, \mathrm{~T}, k_{3}}^{(-1 / 2,-1 / 2)}\left(z_{3}, \bar{z}_{3}\right)\right\rangle=2^{8} \times 5^{2} g_{\mathrm{c}}^{3} \delta\left(\sum_{j} k_{j}\right) .
$$

For untwisted vertices, the correlator is again a lengthy rational expression in $J_{1}, J_{2}, J_{3}$. For $J_{i} \ll \sqrt{1 / \alpha^{\prime}}$, it reduces to

$$
\begin{aligned}
& \left\langle V_{1, k_{1}}^{(-1,-1)}\left(z_{1}, \bar{z}_{1}\right) V_{3, k_{2}}^{(-1 / 2,-1 / 2)}\left(z_{2}, \bar{z}_{2}\right) V_{3, k_{3}}^{(-1 / 2,-1 / 2)}\left(z_{3}, \bar{z}_{3}\right)\right\rangle \\
& \quad=-2^{5} \times 5 \times 59 g_{\mathrm{c}}^{3} \delta\left(\sum_{j} k_{j}\right)+\mathcal{O}\left(J_{i}^{2} \alpha^{\prime}\right) .
\end{aligned}
$$

$\left\langle\boldsymbol{V}_{\mathbf{2}} \boldsymbol{V}_{\mathbf{3}} \boldsymbol{V}_{\mathbf{3}}\right\rangle$. For the correlator $\left\langle V_{2, k_{1}}^{(-1,-1)} V_{3, k_{2}}^{(-1 / 2,-1 / 2)} V_{3, k_{3}}^{(-1 / 2,-1 / 2)}\right\rangle$, the required fermionic correlators are (C.3), (C.5), (C.7). Assembling all terms, and taking into account the asymmetry of the polarization tensor $\alpha$ as well as $k \cdot \alpha_{k}=k \cdot t_{k}=0$, the wanted correlator becomes

$$
\begin{aligned}
& \left\langle V_{2, k_{1}}^{(-1,-1)}\left(z_{1}, \bar{z}_{1}\right) V_{3, k_{2}}^{(-1 / 2,-1 / 2)}\left(z_{2}, \bar{z}_{2}\right) V_{3, k_{3}}^{(-1 / 2,-1 / 2)}\left(z_{3}, \bar{z}_{3}\right)\right\rangle \\
& \quad=-g_{\mathrm{c}}^{3} \delta\left(\sum_{j} k_{j}\right) \alpha_{M N P, \tilde{M} \tilde{N} \tilde{P}}^{k_{1}} t_{\tilde{Q} \tilde{A}, Q A}^{k_{2}} t_{\tilde{R} \tilde{B}, R B}^{k_{3}} X_{\mathrm{L}}^{M N P Q R, A B} X_{\mathrm{R}}^{\tilde{M} \tilde{N} \tilde{P} \tilde{Q} \tilde{R}, \tilde{A} \tilde{B}},
\end{aligned}
$$

where

$$
\begin{gathered}
X_{\mathrm{L}, \mathrm{R}}^{M N P Q R, A B}=\sqrt{2}\left(-\frac{1}{4}\left(\eta^{Q R}-\frac{\alpha^{\prime}}{2} k_{1}^{Q} k_{1}^{R}\right) \Gamma^{M N P} C+\frac{3}{4} \frac{\alpha^{\prime}}{2} k_{1}^{Q} \eta^{M R} \Gamma^{N P} \not k_{3} C\right. \\
\left.+\frac{3}{4} \frac{\alpha^{\prime}}{2} k_{1}^{R} \eta^{M Q} \not k_{2} \Gamma^{N P} C+\frac{1}{8} \frac{\alpha^{\prime}}{2} \not k_{2}\left(-\eta^{Q R} \Gamma^{M N P}+12 \eta^{M R} \eta^{N Q} \Gamma^{P}\right) \not k_{3} C\right)^{A B}
\end{gathered}
$$

are the left/right-moving field contractions. Expanding the polarization tensors, the full correlator can be computed in the same way as for $\left\langle V_{1} V_{3} V_{3}\right\rangle$ above. With twisted polarization tensors, the result is

$$
\left\langle V_{2, \mathrm{~T}, k_{1}}^{(-1,-1)}\left(z_{1}, \bar{z}_{1}\right) V_{3, \mathrm{~T}, k_{2}}^{(-1 / 2,-1 / 2)}\left(z_{2}, \bar{z}_{2}\right) V_{3, \mathrm{~T}, k_{3}}^{(-1 / 2,-1 / 2)}\left(z_{3}, \bar{z}_{3}\right)\right\rangle=-2^{8} \times 3 \times 7 g_{\mathrm{c}}^{3} \delta\left(\sum_{j} k_{j}\right) .
$$


For untwisted vertices the result is a lengthy expression again; for $J_{i} \ll \sqrt{1 / \alpha^{\prime}}$, it becomes

$$
\begin{aligned}
\left\langle V_{2, k_{1}}^{(-1,-1)}\left(z_{1}, \bar{z}_{1}\right) V_{3, k_{2}}^{(-1 / 2,-1 / 2)}\left(z_{2}, \bar{z}_{2}\right)\right. & \left.V_{3, k_{3}}^{(-1 / 2,-1 / 2)}\left(z_{3}, \bar{z}_{3}\right)\right\rangle \\
= & -2^{5} \times 3 \times 5^{2} \times 7 g_{\mathrm{c}}^{3} \delta\left(\sum_{j} k_{j}\right)+\mathcal{O}\left(J_{i}^{2} \alpha^{\prime}\right) .
\end{aligned}
$$

$\langle\boldsymbol{V} \boldsymbol{V} \boldsymbol{V}\rangle$. We now can assemble the correlator of three massive scalars. If we compute the correlators with two or three twisted operators we obtain that all of them vanish, which is accounted for by a supersymmetric Ward identity. For three primaries, the correlator becomes (3.19)

$$
\begin{aligned}
& \left\langle V_{k_{1}}\left(z_{1}, \bar{z}_{1}\right) V_{k_{2}}\left(z_{2}, \bar{z}_{2}\right) V_{k_{3}}\left(z_{3}, \bar{z}_{3}\right)\right\rangle \\
& =g_{\mathrm{c}}^{3} \delta\left(\sum_{j} k_{j}\right) \frac{\Sigma^{4}}{\Delta_{1}^{4} \Delta_{2}^{4} \Delta_{3}^{4}}\left(\frac{1}{2} \Sigma_{2}^{4}+\frac{9}{2} \Sigma_{4}^{2}+\left(2 \Sigma_{2}^{3}-3 \Sigma^{2} \Sigma_{2}^{2}+6 \Sigma^{2} \Sigma_{4}+3 \Sigma_{2} \Sigma_{4}\right) \alpha^{\prime} \Sigma_{4}\right. \\
& \left.\quad+\frac{1}{2}\left(3 \Sigma^{4}+7 \Sigma_{2}^{2}-8 \Sigma^{2} \Sigma_{2}+6 \Sigma_{4}\right)\left(\alpha^{\prime} \Sigma_{4}\right)^{2}-\left(\Sigma^{2}-3 \Sigma_{2}\right)\left(\alpha^{\prime} \Sigma_{4}\right)^{3}+\left(\alpha^{\prime} \Sigma_{4}\right)^{4}\right), \quad(\mathrm{C} .60
\end{aligned}
$$

where

$$
\Sigma_{2}=\alpha_{1} \alpha_{2}+\alpha_{1} \alpha_{3}+\alpha_{2} \alpha_{3}, \quad \Sigma_{4}=\alpha_{1} \alpha_{2} \alpha_{3} \Sigma,
$$

and $\Sigma, \alpha_{j}$ are defined in (1.4). For $J_{i} \ll \sqrt{1 / \alpha^{\prime}}$, the correlator reduces to (1.6)

$$
\left\langle V_{k_{1}}\left(z_{1}, \bar{z}_{1}\right) V_{k_{2}}\left(z_{2}, \bar{z}_{2}\right) V_{k_{3}}\left(z_{3}, \bar{z}_{3}\right)\right\rangle=\frac{3^{8}}{2^{9}} g_{\mathrm{c}}^{3} \delta\left(\sum_{j} k_{j}\right)+\mathcal{O}\left(J_{i}^{2} \alpha^{\prime}\right) .
$$

\section{Mixed correlators}

In the previous section, we checked that all correlators with two or three twisted operators vanish and we also computed the correlators with three untwisted vertices, which correspond to three-point functions of primary operators.

In this section we state the results of correlators with two untwisted vertex operators, which we expect to represent correlation functions of both primary and descendant operators. Starting with the three vertices at the massless level, we have

$$
\left\langle W_{\mathrm{T}, k_{1}}\left(z_{1}, \bar{z}_{1}\right) W_{k_{2}}\left(z_{2}, \bar{z}_{2}\right) W_{k_{3}}\left(z_{3}, \bar{z}_{3}\right)\right\rangle=0 \text {. }
$$

For one massive vertex and two chiral vertices, we also have cancellations for both correlators

$$
\begin{aligned}
& \left\langle W_{k_{1}}\left(z_{1}, \bar{z}_{1}\right) W_{k_{2}}\left(z_{2}, \bar{z}_{2}\right) V_{\mathrm{T}, k_{3}}\left(z_{3}, \bar{z}_{3}\right)\right\rangle=0, \\
& \left\langle W_{\mathrm{T}, k_{1}}\left(z_{1}, \bar{z}_{1}\right) W_{k_{2}}\left(z_{2}, \bar{z}_{2}\right) V_{k_{3}}\left(z_{3}, \bar{z}_{3}\right)\right\rangle=0 .
\end{aligned}
$$

In the case of two massive operators and a massless vertex we get

$$
\begin{aligned}
& \left\langle V_{\mathrm{T}, k_{1}}\left(z_{1}, \bar{z}_{1}\right) V_{k_{2}}\left(z_{2}, \bar{z}_{2}\right) W_{k_{3}}\left(z_{3}, \bar{z}_{3}\right)\right\rangle=0 \\
& \left\langle V_{k_{1}}\left(z_{1}, \bar{z}_{1}\right) V_{k_{2}}\left(z_{2}, \bar{z}_{2}\right) W_{\mathrm{T}, k_{3}}\left(z_{3}, \bar{z}_{3}\right)\right\rangle=-g_{\mathrm{c}}^{3} \delta\left(\sum_{j} k_{j}\right) \frac{\alpha_{3}^{4} \Sigma^{4}}{2 \Delta_{1}^{4} \Delta_{2}^{4}} .
\end{aligned}
$$


Finally, for the case of three massive vertices we have

$$
\left\langle V_{\mathrm{T}, k_{1}}\left(z_{1}, \bar{z}_{1}\right) V_{k_{2}}\left(z_{2}, \bar{z}_{2}\right) V_{k_{3}}\left(z_{3}, \bar{z}_{3}\right)\right\rangle=g_{\mathrm{c}}^{3} \delta\left(\sum_{j} k_{j}\right) \frac{\alpha_{1}^{4} \Sigma^{4}}{2 \Delta_{2}^{4} \Delta_{3}^{4}} .
$$

In section 3 we explained that there cannot be a supercharge that annihilates two untwisted vertex operators, so it might at first seem surprising that four of these correlators actually vanish. Notice though that all of those include a massless vertex operator $W$, which corresponds to a chiral operator. Chiral primaries are annihilated not only by the superconformal charges that in our setup correspond to $Q^{ \pm}$, but also by half of the supergenerators $Q_{\alpha a}$ and $\tilde{Q}_{\dot{\alpha}}^{a}$. Massless fields are then annihilated by an extra eight supercharges, which explains why correlators with at least one twisted vertex and at least one massless operator are indeed vanishing.

Open Access. This article is distributed under the terms of the Creative Commons Attribution License (CC-BY 4.0), which permits any use, distribution and reproduction in any medium, provided the original author(s) and source are credited.

\section{References}

[1] N. Beisert et al., Review of AdS/CFT Integrability: an Overview, Lett. Math. Phys. 99 (2012) 3 [arXiv:1012.3982] [INSPIRE].

[2] N. Gromov, V. Kazakov and P. Vieira, Exact Spectrum of Planar $\mathcal{N}=4$ Supersymmetric Yang-Mills Theory: Konishi Dimension at Any Coupling, Phys. Rev. Lett. 104 (2010) 211601 [arXiv:0906.4240] [INSPIRE].

[3] S. Frolov, Konishi operator at intermediate coupling, J. Phys. A 44 (2011) 065401 [arXiv: 1006.5032] [INSPIRE].

[4] S.S. Gubser, I.R. Klebanov and A.M. Polyakov, Gauge theory correlators from noncritical string theory, Phys. Lett. B 428 (1998) 105 [hep-th/9802109] [INSPIRE].

[5] R. Roiban and A.A. Tseytlin, Quantum strings in $A d S_{5} \times S^{5}$ : strong-coupling corrections to dimension of Konishi operator, JHEP 11 (2009) 013 [arXiv:0906.4294] [INSPIRE].

[6] N. Gromov, D. Serban, I. Shenderovich and D. Volin, Quantum folded string and integrability: from finite size effects to Konishi dimension, JHEP 08 (2011) 046 [arXiv: 1102.1040] [INSPIRE].

[7] M. Beccaria and G. Macorini, Quantum folded string in $S^{5}$ and the Konishi multiplet at strong coupling, JHEP 10 (2011) 040 [arXiv:1108.3480] [INSPIRE].

[8] R. Roiban and A.A. Tseytlin, Semiclassical string computation of strong-coupling corrections to dimensions of operators in Konishi multiplet, Nucl. Phys. B 848 (2011) 251 [arXiv: 1102.1209] [INSPIRE].

[9] M. Beccaria, S. Giombi, G. Macorini, R. Roiban and A.A. Tseytlin, 'Short' spinning strings and structure of quantum AdS $S_{5} \times S^{5}$ spectrum, Phys. Rev. D 86 (2012) 066006 [arXiv: 1203.5710] [INSPIRE].

[10] M. Beccaria and A.A. Tseytlin, More about 'short' spinning quantum strings, JHEP 07 (2012) 089 [arXiv: 1205.3656] [INSPIRE].

[11] B. Basso, Exciting the GKP string at any coupling, Nucl. Phys. B 857 (2012) 254 [arXiv: 1010.5237] [INSPIRE]. 
[12] N. Gromov and S. Valatka, Deeper Look into Short Strings, JHEP 03 (2012) 058 [arXiv:1109.6305] [INSPIRE].

[13] S. Frolov, M. Heinze, G. Jorjadze and J. Plefka, Static gauge and energy spectrum of single-mode strings in $A d S_{5} \times S^{5}$, J. Phys. A 47 (2014) 085401 [arXiv:1310.5052] [INSPIRE].

[14] J. Escobedo, N. Gromov, A. Sever and P. Vieira, Tailoring Three-Point Functions and Integrability, JHEP 09 (2011) 028 [arXiv:1012.2475] [INSPIRE].

[15] J. Escobedo, N. Gromov, A. Sever and P. Vieira, Tailoring Three-Point Functions and Integrability II. Weak/strong coupling match, JHEP 09 (2011) 029 [arXiv:1104.5501] [INSPIRE].

[16] N. Gromov, A. Sever and P. Vieira, Tailoring Three-Point Functions and Integrability III. Classical Tunneling, JHEP 07 (2012) 044 [arXiv:1111.2349] [INSPIRE].

[17] I. Kostov, Three-point function of semiclassical states at weak coupling, J. Phys. A 45 (2012) 494018 [arXiv: 1205.4412] [inSPIRE].

[18] O. Foda, Y. Jiang, I. Kostov and D. Serban, A tree-level 3-point function in the SU(3)-sector of planar $N=4$ SYM, JHEP 10 (2013) 138 [arXiv:1302.3539] [INSPIRE].

[19] K. Zarembo, Holographic three-point functions of semiclassical states, JHEP 09 (2010) 030 [arXiv:1008.1059] [INSPIRE].

[20] M.S. Costa, R. Monteiro, J.E. Santos and D. Zoakos, On three-point correlation functions in the gauge/gravity duality, JHEP 11 (2010) 141 [arXiv:1008.1070] [INSPIRE].

[21] J.A. Minahan, Holographic three-point functions for short operators, JHEP 07 (2012) 187 [arXiv:1206.3129] [INSPIRE].

[22] J. Polchinski, $S$ matrices from AdS space-time, hep-th/9901076 [INSPIRE].

[23] L. Susskind, Holography in the flat space limit, hep-th/9901079 [INSPIRE].

[24] V. Balasubramanian, S.B. Giddings and A.E. Lawrence, What do CFTs tell us about Anti-de Sitter space-times?, JHEP 03 (1999) 001 [hep-th/9902052] [INSPIRE].

[25] M. Bianchi, J.F. Morales and H. Samtleben, On stringy $A d S_{5} \times S^{5}$ and higher spin holography, JHEP 07 (2003) 062 [hep-th/0305052] [INSPIRE].

[26] N. Beisert, M. Bianchi, J.F. Morales and H. Samtleben, On the spectrum of AdS/CFT beyond supergravity, JHEP 02 (2004) 001 [hep-th/0310292] [INSPIRE].

[27] R.H. Boels, Three particle superstring amplitudes with massive legs, JHEP 06 (2012) 026 [arXiv:1201.2655] [INSPIRE].

[28] S. Lee, S. Minwalla, M. Rangamani and N. Seiberg, Three point functions of chiral operators in $D=4, N=4 S Y M$ at large- $N$, Adv. Theor. Math. Phys. 2 (1998) 697 [hep-th/9806074] [INSPIRE].

[29] T. Klose and T. McLoughlin, A light-cone approach to three-point functions in $A d S_{5} \times S^{5}$, JHEP 04 (2012) 080 [arXiv:1106.0495] [INSPIRE].

[30] E. Witten, Anti-de Sitter space and holography, Adv. Theor. Math. Phys. 2 (1998) 253 [hep-th/9802150] [INSPIRE].

[31] D.Z. Freedman, S.D. Mathur, A. Matusis and L. Rastelli, Correlation functions in the CFT(d)/AdS(d+1) correspondence, Nucl. Phys. B 546 (1999) 96 [hep-th/9804058] [INSPIRE].

[32] E.I. Buchbinder and A.A. Tseytlin, Semiclassical correlators of three states with large $S^{5}$ charges in string theory in $A d S_{5} \times S^{5}$, Phys. Rev. D 85 (2012) 026001 [arXiv:1110.5621] [INSPIRE]. 
[33] D. Friedan, E.J. Martinec and S.H. Shenker, Conformal Invariance, Supersymmetry and String Theory, Nucl. Phys. B 271 (1986) 93 [inSPIRE].

[34] I.G. Koh, W. Troost and A. Van Proeyen, Covariant Higher Spin Vertex Operators in the Ramond Sector, Nucl. Phys. B 292 (1987) 201 [INSPIRE].

[35] N. Beisert, BMN operators and superconformal symmetry, Nucl. Phys. B 659 (2003) 79 [hep-th/0211032] [INSPIRE].

[36] J.A. Minahan and K. Zarembo, The Bethe ansatz for $N=4$ super Yang-Mills, JHEP 03 (2003) 013 [hep-th/0212208] [INSPIRE].

[37] E. D'Hoker, D.Z. Freedman, S.D. Mathur, A. Matusis and L. Rastelli, Extremal correlators in the AdS/CFT correspondence, hep-th/9908160 [INSPIRE].

[38] R.A. Janik, P. Surowka and A. Wereszczynski, On correlation functions of operators dual to classical spinning string states, JHEP 05 (2010) 030 [arXiv: 1002.4613] [INSPIRE].

[39] E. D'Hoker, D.Z. Freedman, S.D. Mathur, A. Matusis and L. Rastelli, Graviton exchange and complete four point functions in the AdS/CFT correspondence, Nucl. Phys. B 562 (1999) 353 [hep-th/9903196] [InSPIRE].

[40] E. D'Hoker, D.Z. Freedman and L. Rastelli, AdS/CFT four point functions: how to succeed at $z$ integrals without really trying, Nucl. Phys. B 562 (1999) 395 [hep-th/9905049] [INSPIRE].

[41] J. Penedones, Writing CFT correlation functions as AdS scattering amplitudes, JHEP 03 (2011) 025 [arXiv:1011.1485] [INSPIRE].

[42] A.L. Fitzpatrick, J. Kaplan, J. Penedones, S. Raju and B.C. van Rees, A Natural Language for AdS/CFT Correlators, JHEP 11 (2011) 095 [arXiv:1107.1499] [INSPIRE].

[43] M.S. Costa, V. Goncalves and J. Penedones, Conformal Regge theory, JHEP 12 (2012) 091 [arXiv: 1209.4355] [INSPIRE].

[44] G. Arutyunov and S. Frolov, On String S-matrix, Bound States and TBA, JHEP 12 (2007) 024 [arXiv:0710.1568] [INSPIRE].

[45] G. Arutyunov and S. Frolov, Thermodynamic Bethe Ansatz for the $A d S_{5} \times S^{5}$ Mirror Model, JHEP 05 (2009) 068 [arXiv:0903.0141] [INSPIRE].

[46] N. Gromov, V. Kazakov and P. Vieira, Exact Spectrum of Anomalous Dimensions of Planar $N=4$ Supersymmetric Yang-Mills Theory, Phys. Rev. Lett. 103 (2009) 131601 [arXiv:0901.3753] [INSPIRE].

[47] D. Bombardelli, D. Fioravanti and R. Tateo, Thermodynamic Bethe Ansatz for planar AdS/CFT: a Proposal, J. Phys. A 42 (2009) 375401 [arXiv:0902.3930] [inSPIRE].

[48] N. Gromov, V. Kazakov, S. Leurent and D. Volin, Solving the AdS/CFT Y-system, JHEP 07 (2012) 023 [arXiv: 1110.0562] [INSPIRE].

[49] N. Gromov, V. Kazakov, S. Leurent and D. Volin, Quantum spectral curve for $A d S_{5} / C F T_{4}$, Phys. Rev. Lett. 112 (2014) 011602 [arXiv:1305.1939] [INSPIRE].

[50] J.A. Polchinski, String Theory, Cambridge University Press, Cambridge, U.K. (1998)

[51] V.A. Kostelecky, O. Lechtenfeld, W. Lerche, S. Samuel and S. Watamura, Conformal Techniques, Bosonization and Tree Level String Amplitudes, Nucl. Phys. B 288 (1987) 173 [INSPIRE].

[52] W.-Z. Feng, D. Lüst and O. Schlotterer, Massive Supermultiplets in Four-Dimensional Superstring Theory, Nucl. Phys. B 861 (2012) 175 [arXiv:1202.4466] [INSPIRE]. 